\title{
Evidences for a Unified Physics, in Full Accordance with the Newtonian Laws
}

\author{
Alfredo Bacchieri \\ University of Bologna, Bologna, Italy \\ Email: abacchieri@libero.it
}

How to cite this paper: Bacchieri, A. (2016) Evidences for a Unified Physics, in Full Accordance with the Newtonian Laws. Journa of Modern Physics, 7, 2231-2255. http://dx.doi.org/10.4236/jmp.2016.715193

Received: August 3, 2016

Accepted: November 27, 2016

Published: November 30, 2016

Copyright $\odot 2016$ by author and Scientific Research Publishing Inc. This work is licensed under the Creative Commons Attribution International License (CC BY 4.0).

http://creativecommons.org/licenses/by/4.0/

\section{(c) (i) Open Access}

\begin{abstract}
We show that the speed of a longitudinal-extended, elastic (variable length), and massive particle, emitted by a source during an emission time $T$, at speed $u$ (escape speed from all the masses in space), is invariant for every real measurement, (intending a measurement requiring an interaction light-matter), in spite of any reciprocal motion source-Observer. Thus we may argue that the light has to be composed of such particles (photons) moving at speed $c=u$. Compliance of these photons with Newtonian mechanics is shown for many effects, (like the Doppler effect, redshift, time dilation, etc.), highlighting the differences versus the Relativity. In the $2^{\text {nd }}$ part, on the assumption that the electron charge can be considered as a point-particle fixed to the electron surface, always facing the atom nucleus during the electron revolution, we revised the light-matter interaction, showing that it only depends on the particular impacts between these photons and the circling electrons: for instance, on $\mathrm{H}$ atom, we found 137 circular orbits only, the last one being the ionization orbit, where the electron orbital speed becomes $v_{\mathrm{i}}=c / 137^{2}$. [Classical mechanics implies that orbiting electrons produce an electro-magnetic radiation causing their fall into the nucleus: on Section 3.5, the reason why the electron circular orbits are stable].
\end{abstract}

\section{Keywords}

Doppler Effect for the Light, Harvard Tower Experiment, Gravitational Redshift, Time Dilation, Compton Effect

\section{Introduction}

This paper is based on following assumptions:

I. Finite mass of the universe, (implying a finite value of the total gravitational potential $U$ ). 
II. Light composed of longitudinal-extended elastic particles moving at speed $c=u$. On above bases we obtain, among others, these following results:

a) The relation $u=(-2 U)^{1 / 2}$, where $u$ is total escape speed, that is the escape speed from all the masses (in space); then we assumed $c=u$.

b) The measured constancy of $c$, under a constant potential $U$, (like on Earth), for every Observer.

c) Doppler effect (DE) equations for the light, slightly different from the relativistic ones.

d) Regarding the Harvard Tower Experiment, the compensating velocity, (to restore the resonance source-absorber), see Section 2.4, has same value but contrary direction with respect to the one predicted by the Relativity.

e) On Earth, at height $h$, (e.g. at GPS satellites level), it is shown that a source (of light) emits at a lower frequency, inducing atomic clocks to run faster.

f) High redshifts, related to far sources, depend on the increase of $c$ (as well as the increase of the photons length $\lambda$ ) during the path of light toward the Earth, (where $\left.\left|U_{\mathrm{o}}\right| \gg\left|U_{\rightarrow \infty}\right|\right)$.

In the $2^{\text {nd }}$ part, we show the interaction between our particles and circling electrons; we revised the electron structure, assuming the electron charge as a point-particle, (facing the atom nucleus during the electron orbit), which turns out to be the impact point between photons and electron; some results:

g) On $\mathrm{H}$ atom there are only $n=1,2, \cdots, 137$ electron circular orbits; along each of them, $n$ is also the number of admitted photons. We show that

$2 v_{0} / v_{\mathrm{e} 0}=\alpha\left(c m_{\mathrm{r}} / 2 h R_{\mathrm{H}}\right)^{1 / 2}=1$ (exactly) with $v_{0}$ the photons admitted frequency along the ground-state orbit $r_{0}$ (different, because of our new atom structure, from the Bohr radius) and $v_{\mathrm{e} 0}$ the frequency of the electron reduced mass $m_{\mathrm{r}}$ along $r_{0}$, with $\alpha$ the fine structure constant.

h) On Photoelectric effect, the number of photons hitting the electron varies from $n_{\mathrm{f}}$ to $n_{\mathrm{f}}^{1 / 2}$ where $n_{\mathrm{f}}$ is related to the specific threshold frequency $v_{\mathrm{f}}\left(=W_{\mathrm{f}} / h\right)$. For instance, it is shown that, as for Caesium (having $W_{\mathrm{f}} \cong 2 \mathrm{eV}$ ), one gets $n_{\mathrm{f}}=361$.

i) On Compton Effect, the number of photons admitted is one We point out that we got the Compton equation via our Doppler effect for the light, different from the relativistic Doppler Effect.

\section{Part 1}

\subsection{Total Escape Speed}

This argument has been widely treated on Section 2 of our previous paper [1]. Here we remember:

$$
u=\sqrt{-2 U}=\sqrt{2 M G / s}
$$

escape speed of a particle $m$ at a distance $s$ from the mass $M$, with $U$ the gravitational potential acting on $m$; if $M$ is a real mass, $s$ becomes the distance $m-\mathrm{C}_{\mathrm{p}}$ with $\mathrm{C}_{\mathrm{p}}$ the Centre of Potential of $M$, that is the point where $|U|$ has the max value), while the es- 
cape velocity of $m$ which has to be referred to $\mathrm{C}_{\mathrm{p}}$,

$$
\mathbf{v}_{\mathrm{Cpm}}=\mathbf{u}
$$

may be called as absolute escape velocity of $m$, absolute as referred to $\mathrm{C}_{\mathrm{p}}$; then

$$
U_{1,2} \equiv U_{1}+U_{2}=\left(-M_{1} G / s_{1}\right)+\left(-M_{2} G / s_{2}\right)
$$

is the potential due to two masses; then the escape speed from two masses becomes

$$
u_{1,2} \equiv \sqrt{-2 U_{12}}=\sqrt{\left(2 M_{1} G / s_{1}\right)+\left(2 M_{2} G / s_{2}\right)}
$$

yielding

$$
u_{1,2}^{2}=u_{1}^{2}+u_{2}^{2}
$$

thus

$$
u=\sqrt{\sum u_{n}^{2}}=\sqrt{-2 U}=\sqrt{2\left(\sum M_{n} G / s_{n}\right)}
$$

is the total escape speed, with $U$ the total potential; $\mathbf{u}$ (absolute escape velocity)has to be referred to the centre of potential of all the masses, $C_{p}$. Now, if $S$ is a Source emitting a particle $m$, we may call

$$
\mathbf{v}_{\mathrm{S} m}=\mathbf{u}
$$

as relative escape velocity of $m$ from $S$, (relative as $\mathbf{u}$ is referred to $S$ ). We assume now

$$
c \equiv u=\sqrt{-2 U}
$$

showing that $c$ depends on $U$; on Earth, $U$ is practically constant, see the final "Conclusions".

Equation (8) is supported by a cosmological reason, as better explained between the Equation (5) and Equation (6) on [1]: in short, $c>u$ implies the masses dispersion, $c<$ $u$ implies a gravitational collapse, where as $c=u$, appears to be the right speed of the light to avoid the two said events (collapse or dispersion).

\subsection{Invariance of $c$ for Every Observer, Under the Newtonian Laws}

-This chapter is a deep revision/improvement of chapter4 of our previous paper [1]-

We assume now the light as composed of particular particles (photons), giving a Newtonian reason to the (apparent) constancy of $c$, defined as follows:

"Longitudinally-extended, elastic and massive particles having speed equal to the total escape speed $u$, and moving along rays, (continuous succession of photons)".

(More photons emitted by a source, during an emission time $T$, need an equal number of rays).

Along each ray, every tail of a photon corresponds to the front of the next photon.

Now, on Figure 1, let $S$ be a Source, (moving from an Observer $O$ with velocity $\mathbf{v}_{\mathrm{OS}}$ ), starting to emit, at $t=0$, (when $\mathrm{S}$ is coincident with $\mathrm{O}$ ), a photon (with front $\mathrm{A}$ ), along the direction S-O; therefore

$$
\mathbf{v}_{\mathrm{OA}}=\mathbf{u}
$$

represents, see Equation (7), at $t=0$, the relative escape velocity of the front $\mathrm{A}$ from $\mathrm{O}$, 
while the velocity of the front $A$, with respect to $S$, that is $\mathbf{v}_{S A}$, turns out to be

$$
\mathbf{v}_{\mathrm{SA}}=\mathbf{v}_{\mathrm{SO}}+\mathbf{v}_{\mathrm{OA}}=\mathbf{u}-\mathbf{v}_{\mathrm{OS}}
$$

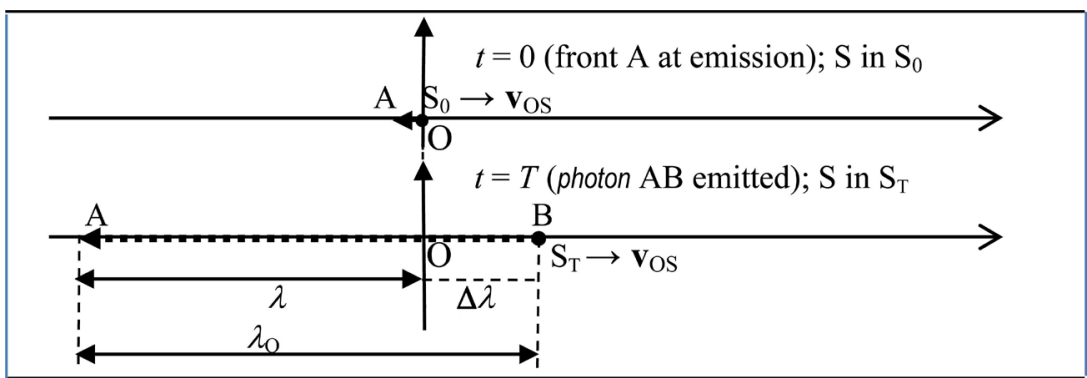

Figure 1. The source $\mathrm{S}$, in motion from the observer $\mathrm{O}$, emits the photon $\mathrm{AB}$.

Now, being $\mathrm{S}_{\mathrm{T}}$ be the position of $\mathrm{S}$ at $t=T$, we get

$$
\lambda_{\mathrm{O}}=\mathbf{v}_{\mathrm{SA}} T=\left(\mathbf{u}-\mathbf{v}_{\mathrm{OS}}\right) T=\lambda-\mathbf{v}_{\mathrm{OS}} T
$$

where $\boldsymbol{\lambda}_{\mathrm{O}}$ is the photon $\mathrm{AB}$ emitted with $\mathrm{S}$ in motion from $\mathrm{O}$, whereas $\boldsymbol{\lambda}(\equiv \mathbf{u} T$ ) would be the photon $\mathrm{AO}$ if, during $T, \mathrm{v}_{\mathrm{OS}}=0$. Thus, at $t=T$, if the source is receding from the front A, as in Figure 1, the photon length $\lambda_{\mathrm{O}}$, (for the Observer O), from Equation (11), becomes

$$
\lambda_{\mathrm{O}}=u T+v_{\mathrm{OS}} T=\lambda_{\mathrm{O}}+\lambda=\lambda(1+\beta),\left(\text { with } \beta=v_{\mathrm{OS}} / u\right)
$$

where $v_{\mathrm{OS}}=\left|\mathbf{v}_{\mathrm{OS}}\right|$, with $\Delta \lambda\left(=v_{\mathrm{OS}} T\right)$ the path O-S $\mathrm{T}_{\mathrm{T}}$ covered by S during $T$. For instance, if $v_{\mathrm{OS}}=0$, the (12) gives $\lambda_{\mathrm{O}}=u T=\lambda$ with $\lambda$ the photon length as seen by $\mathrm{S}$.

The Equation (12) shows that the length of a photon during its emission, given $u$ and $T$, depends on $\mathbf{v}_{\mathrm{OS}}$, meaning that Observers in reciprocal motion state different length for the same photon.

Now, referring to an Observer O, the speed of a photon, (since its length could vary), has to be defined considering its length $\lambda_{\mathrm{O}}$ referred to its transit time $T_{\mathrm{O}}$, leading to

$$
u_{\mathrm{O}} \equiv c_{\mathrm{O}}=\left|\lambda-\mathbf{v}_{\mathrm{OS}} T\right| / T_{\mathrm{O}}=\lambda_{\mathrm{O}} / T_{\mathrm{O}}
$$

Returning to Figure 1, the transit time $T_{\mathrm{O}}$ of the photon $\mathrm{AB}$, for the Observer $\mathrm{O}$, is given by the time the front A needs to cover the path $\lambda$, that is $T(=\lambda / u)$, plus the time the tail $\mathrm{B}$ needs to cover the path $\mathrm{S}_{\mathrm{T}}-\mathrm{O}=\Delta \lambda\left(=v_{\mathrm{OS}} T\right)$ which is $\Delta T=v_{\mathrm{OS}} T / u$, giving

$$
T_{\mathrm{O}}=T+\Delta \lambda / u=T+v_{\mathrm{OS}} T / u=T(1+\beta) \quad\left(\text { with } \beta=v_{\mathrm{OS}} / u\right)
$$

Thus, see Equation (13), referring to the Observer $\mathrm{O}$, the speed of the photon $\mathrm{AB}$ becomes

$$
u_{\mathrm{O}}=\lambda_{\mathrm{O}} / T_{\mathrm{O}}=\lambda(1+\beta) / T(1+\beta)=\lambda / T(\equiv c)
$$

showing that $c$ is invariant, under the Newtonian laws, in spite of any speed SourceObserver.

Anyhow, a photon, once emitted, has a constant length for every Observer, hence its speed has to vary for Observers in reciprocal motion, but we point out that the measurements of $c$ via the method $d / t$ implies that the light has to cross (or to be reflected 
by) an Observer $\mathrm{O}$ (taking the initial time); this means that $\mathrm{O}$ becomes the source $\mathrm{S}$ of photons emitted by a source at rest with $\mathrm{O}\left(v_{\mathrm{OS}}=0\right)$ who will state a length $\lambda_{\mathrm{O}}=\lambda$, a transit time $T_{\mathrm{O}}=T$ giving to $c$ a constant measured value.

Along one ray, the frequency of photons, for an Observer $\mathrm{O}$, is their number crossing O during a time $t$, that is $v=n / t$, thus for $t=T_{\mathrm{O}}$, (transit time of one photon, for an Observer O), from Equation (14),

$$
v_{\mathrm{O}}\left(=1 / T_{\mathrm{O}}\right)=1 / T(1 \pm \beta)=v /(1 \pm \beta)
$$

with the sign + when $\mathrm{S}$ and $\mathrm{O}$, during the emission, are receding from each other. We point out that for $\beta=0,\left(v_{\mathrm{OS}} / u=0\right)$, the photons frequency as stated by a source $\mathrm{S}\left(v_{\mathrm{s}}\right)$, has to be equal to the one stated by $\mathrm{O}\left(v_{\mathrm{O}}\right)$, which is also valid if $\mathrm{O}$ and $\mathrm{S}$ belong to different potential, (e.g., the equality of the number of balls falling from the top of a tower with respect to an Observer at the tower base), and this, for $v_{\mathrm{OS}}=0$, always implies $v_{\mathrm{s}}=$ $v_{\mathrm{O}}$. Figure 1, as well as Equations ((12) and (16)) represent the longitudinal Doppler effect for the light while, in general, this effect, with $\alpha$ the angle between the direction of $\mathrm{S}$ and $\mathrm{OS}$, (and with $\mathrm{S}$ receding from $\mathrm{O}$ ), see Figure 2, can be written as

$$
\lambda_{\mathrm{O}}=\lambda+v_{\mathrm{OS}} T \cos \alpha=\lambda(1+\beta \cos \alpha) \quad \text { (with } \beta=v_{\mathrm{OS}} / c \text { ) }
$$

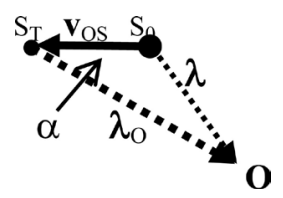

Figure 2. Doppler effect.

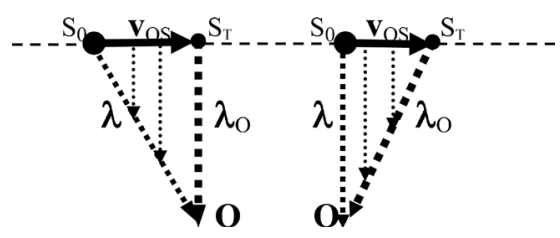

Figure 3. Transverse doppler effect.

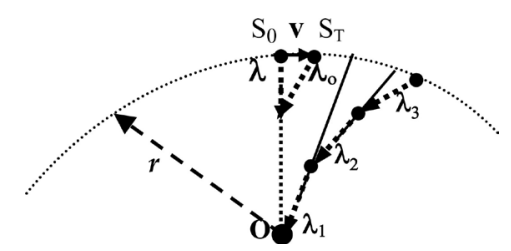

Figure 4. Source circling around the observer O.

As for the Transverse Doppler effect, see Figure 3, in general, we have

$$
\lambda_{\mathrm{O}}=\sqrt{\lambda^{2} \pm\left(v_{\mathrm{OS}} T\right)^{2}}=\lambda \sqrt{1 \pm \beta^{2}}
$$

As for a source circling around an Observer $\mathrm{O}$, see Figure 4, it is always $\lambda_{\mathrm{O}}>\lambda$ as follows:

$$
\lambda_{\mathrm{O}}=\sqrt{\lambda^{2}+\left(v_{\mathrm{OS}} T\right)^{2}}=\lambda \sqrt{1+\beta^{2}}=\lambda \sqrt{1+\omega^{2} r^{2} / c^{2}}
$$


while their photons transit time is

$$
T_{\mathrm{O}}=T \sqrt{1+\beta^{2}}=T \sqrt{1+\omega^{2} r^{2} / c^{2}}
$$

where $r$ is the orbit radius, $\omega$ the angular speed, yielding, to every photon, the speed $c_{\mathrm{O}}$ $=c$.

\subsection{Physical Characteristics of These Photons}

Also this argument has been widely treated on our previous paper [1]: here we point out that as the energy of light $E=m c^{2}$ is valid for any mass, it has to be also valid for the light (massive on our bases) and therefore, writing $E=\frac{1}{2} m c^{2}+\frac{1}{2} m c^{2}$ we have to argue that each photon is provided with an internal energy $K_{\mathrm{i}}=\frac{1}{2} m c^{2}$ equal to its kinetic energy $K_{c}$. Toward the infinity, (where $c_{\infty} \rightarrow 0$ ) both $K_{\mathrm{c}}$ and $K_{\mathrm{i}} \rightarrow 0$ and therefore, since $E=h v$,

$$
E=K_{\mathrm{c}}+K_{\mathrm{i}}=\frac{1}{2} m c^{2}+\frac{1}{2} m c^{2}=m c^{2}=h v
$$

represents the energy of one ray of light, (where photons are flowing) and where

$$
m=h v / c^{2} \equiv \gamma v(\mathrm{~kg})
$$

is the mass of light, having frequency $v$, passing along one ray in $1 \mathrm{~s}$, while the constant

$$
\gamma \equiv h / c^{2}=m / v=7.372495 \times 10^{-51} \mathrm{~kg} \cdot \mathrm{s}
$$

where

$$
h=\gamma c^{2}=m c^{2} T
$$

thus the Planck's constant turns out to be the energy of one photon.

Now, since $m$ is the mass of light passing along one ray in $1 \mathrm{~s}$, the term $n_{\mathrm{r}} m c^{2}$, with $n_{\mathrm{r}}$ the number of rays emitted by a source $S$, becomes the energy emitted by $S$ in $1 \mathrm{~s}$; this unitary (unit of time) energy shall be equal to the supplied power $P$ during 1s,yielding

$$
n_{\mathrm{r}} m c^{2}=P,
$$

therefore

$$
m_{\mathrm{tot}}=n_{\mathrm{r}} m=P / c^{2}
$$

is the total mass lost per second by a source of light; e.g. for a $1 \mathrm{~W}$ lamp, we get $m_{\text {tot }}=P / c^{2} \cong 1.1 \times 10^{-17} \mathrm{~kg} \cdot \mathrm{s}^{-1}$, while the number $n_{\mathrm{r}}$ of rays is

$$
n_{\mathrm{r}}\left(=m_{\mathrm{tot}} / \gamma v\right)=P / c^{2} \gamma v=P / h v
$$

in our case, $n_{\mathrm{r}} \cong 3 \times 10^{18}$ rays. Then, for a given power $P$, the higher is the frequency, the lower is the number of rays, as shown by (27) written as $n_{\mathrm{r}} v=P / h$. The number of photons emitted in $1 \mathrm{~s}$ is

$$
n_{\gamma}\left(=n_{\mathrm{r}} v\right)=P v / h v=P / h
$$

which, for $P=1 \mathrm{~W}$, gives $n_{\gamma}=h^{-1}\left(=1.5 \times 10^{33}\right.$ photons $\left./ \mathrm{s}\right)$, thus the inverse of Planck's constant turns out to be the number of photons emitted in 1s by a source of unitary 
power, and this great number of photons allows the light to be treated as a wave.

Now, during inelastic impacts, (like on absorption or photoelectric effetcs), both kinetic and internal energy of the light are involved, so the momentum transferred to the electron is

$$
\mathbf{p}=2 m \mathbf{c}=2 \gamma v \mathbf{c}=2 \gamma \mathbf{c} / T
$$

while, during elastic impacts, the momentum transferred to the electron is

$$
\mathbf{p}=m^{\prime} \mathbf{c}=\gamma v^{\prime} \mathbf{c}=\gamma \mathbf{c} / T^{\prime}
$$

either for incident or for reflected photons, with $T$ the total impact time for this interaction, as we show on Section 3.7, Compton effect; at this regard, via the (30), as well as via our Doppler effect equation, see (12), we get the Compton equation, which can not be obtained by the Relativity via their Doppler effect equations.

\subsection{Re-Visitation of the Harvard Tower Experiment (HTE), Time Dilation, Gravitational Redshift}

Also this argument has been widely treated on our previous paper [1]. Here the main results: referring to Figure $5(\mathrm{a})$, where $h$ is the tower height, and $M_{\mathrm{E}}$ is the mass of Earth, writing the Equation (8) as $c^{2}=-2 U$, we can obtain

$$
\left.\left(c_{h}-c_{0}\right) / c_{0}=-\Delta U / c_{0}^{2}=-M_{\mathrm{E}} G h / r_{h} r_{0} c_{0}^{2} \cong-g h / c_{0}^{2} \text { (valid for } r_{h} \cong r_{0}\right)
$$

that is the variation of $c$ from the tower base to its top, where $c_{0}$ and $c_{h}$ are the corresponding values of $c, \Delta U\left(=U_{\mathrm{E} h}-U_{\mathrm{E} 0}\right)$ is the variation of potential; hence $c_{h}<c_{0}$, with $c_{h}=c_{0}\left(1-g h / c_{0}^{2}\right)$.

Let now $\mathrm{S}$ be a Mossbauer source and $\mathrm{A}$ a related absorber, both, for instance, at the tower base, therefore in resonance. Then, see Figure 5(b), taking A to the top, and since $\mathrm{A}$ and $\mathrm{S}$ are now at rest, the frequency of the photons emitted by $\mathrm{S}$, has to be equal to the one observed by A, that is $v_{h}=v_{0}$, therefore the Equation (31), as for the photons emitted by $S$, can be written as

$$
\Delta c / c_{0}=\Delta \lambda / \lambda_{0}=-g h / c_{0}^{2}
$$

and since $c_{h}<c_{0}$, it must be $\lambda_{h}<\lambda_{0}$, so, contrary to Relativity, A observes a blue-shift effect.

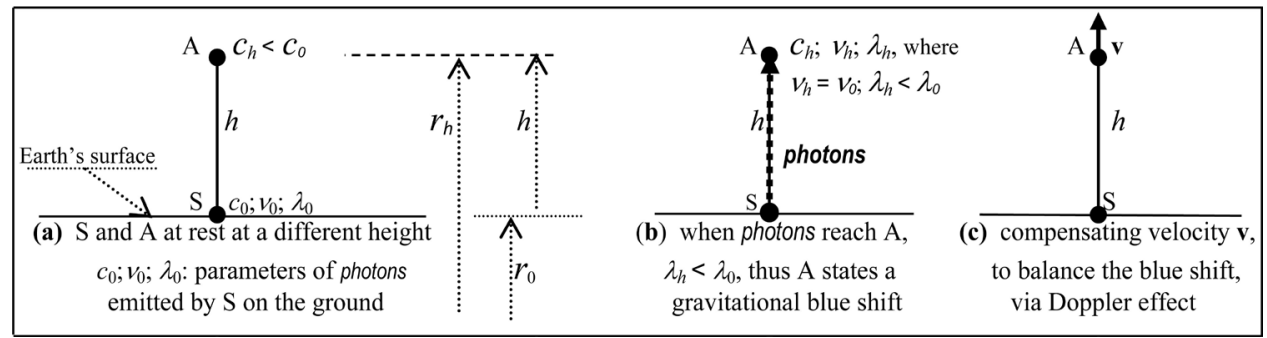

Figure 5. Harvard tower experiment (HTE) scheme; source at the base, our results.

Thus, to restore the resonance via Doppler Effect (DE), S and A, see Figure 5(c), have to recede from each other, in order that the photon length should increase, see 
Equation (12), from $\lambda_{h}$ to $\lambda_{0}=\lambda_{h}(1+\beta)$ with $\beta=v_{\mathrm{AS}} / c \equiv v / c$, so, equating the photon length variation $(\Delta \lambda / \lambda=-v / c$ due, see (17), to $\mathrm{DE})$, to $\Delta \lambda / \lambda$, due to the altitude, as given by (32), we get $v / c=g h / c_{0}^{2}$, yielding

$$
v=g h / c_{0}=7.5 \times 10^{-7} \mathrm{~m} / \mathrm{s} \quad(\text { for } h=22.5 \mathrm{~m}) .
$$

This value is also predicted by GR which, implying a decrease of $v$ for the light moving from the base to the top, predicts an opposite direction of $\mathbf{v}$ with respect to the one shown on Figure 5(c); at this regard, Pound-Rebka and Pound-Snider, [2] [3] [4], gave no clear indication about the direction of the compensating velocity.

Moreover, see Figure 5(b), with $S$ on the base, emitting upward, A goes out of resonance and since on our bases $v_{h}=v_{0}$, the non-resonance is physically related to a variation of $\lambda$.

Now, see Figure 6(a), with $A$ on the base, taking $S$ to the top, the experience shows that the absorber goes out of resonance. Indeed, with $S$ on the top, the (31) shows $c_{h}<$ $c_{0}$, but what about the initial parameters of the photons emitted in altitude, $v_{h}$ and $\lambda_{h}$ ?

Well, see Figure 6(b), since $S$ and $A$ are at reciprocal rest, the frequency of the photons arriving to the base, is $v_{h-0}=v_{h}$, hence along the path top-base, the photon length $\lambda_{h-0}$ has to increase (following the increase of $c$ from $c_{h}$ to $c_{0}$ ); thus we have to argue that taking the source on top, see Figure 6(a), the photons initial length must be $\lambda_{h}=\lambda_{0}$, in order that after the path top-base, it becomes $\lambda_{h-0}>\lambda_{0}$ (inducing the absorber to go out of resonance).This implies

$$
v_{h}=c_{h} / \lambda_{h}=c_{0}\left(1-g h / c_{0}^{2}\right) / \lambda_{0}=v_{0}\left(1-g h / c_{0}^{2}\right)=v_{0}\left(1-\Delta U / c_{0}^{2}\right)
$$

showing that, taking $S$ to the top, $v_{h}<v_{0}$. Now, along the path top-base, $\Delta \lambda / \lambda$ has opposite sign with respect to (32), yielding $\left(\lambda_{h-0}-\lambda_{h}\right) / \lambda_{h}=g h / c_{0}^{2}$, and since $\lambda_{h}=\lambda_{0}$, we get

$$
\lambda_{h-0}=\lambda_{h}\left(1+g h / c_{0}^{2}\right)=\lambda_{0}\left(1+g h / c_{0}^{2}\right)
$$

showing an increase of $\lambda$ from the top to the base. Hence the absorber, on the base, will state a red-shift so, to compensate it via Doppler shift, see Figure 6(c), S and A have now to move relative to each other; on the contrary, according to Relativity, $A$ and $S$ should recede from each other.

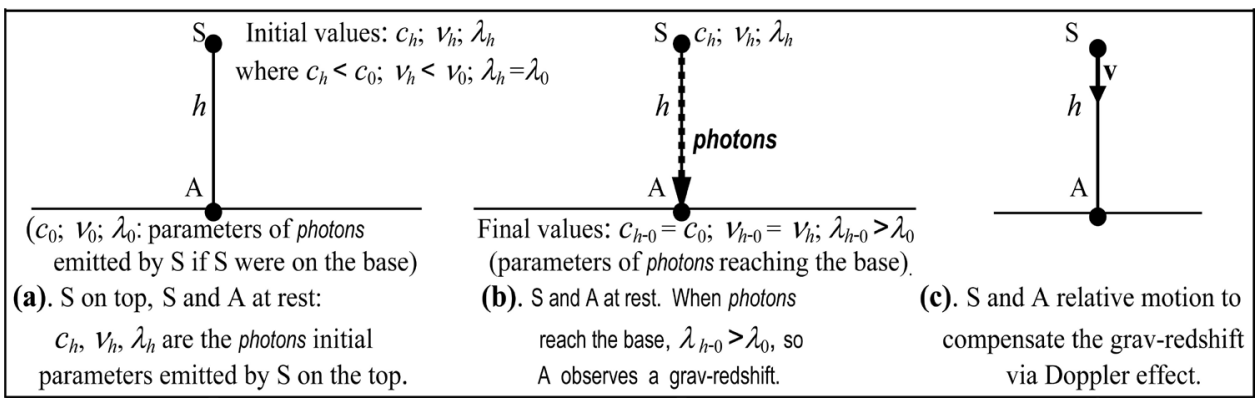

Figure 6. Harvard tower experiment scheme; source on the top, our results.

Time dilation: Atomic clocks in altitude ( $h$-clocks) are ticking faster than identical clocks on the ground (g-clocks): indeed, on our bases, at height $h$, see (30), we have 
$\left(c_{h}-c_{0}\right) / c_{0}=-\Delta U / c_{0}^{2}$, while taking a clock to a GPS satellite, see also Figure 6(a), from (34), one finds

$$
T_{h}=T_{0} /\left(1-\Delta U / c_{0}^{2}\right)
$$

with $T_{h}=1 / v_{h}$ the counted time of one photon emitted by a $h$-clock, while $T_{0}$ to a g-clock. Thus the variation of the counted time between the two clocks, for every emitted photon, becomes

$$
T_{\mathrm{ph}}=T_{h}-T_{0}=\left[T_{0} /\left(1-\Delta U / c_{0}^{2}\right)\right]-T_{0}=\left(T_{0} \Delta U / c_{0}^{2}\right) /\left(1-\Delta U / c_{0}^{2}\right)
$$

Now, the frequency of photons is their number emitted in $1 \mathrm{~s}$ along one ray, therefore the term

$$
n_{1 \mathrm{~s}} \equiv v_{0} t_{1 \mathrm{~s}} \quad\left(\text { with } t_{1 \mathrm{~s}}=1 \mathrm{~s}\right)
$$

is the number of photons (atomic transition of Cs 137) that constitute a one-second, so that

$$
v_{h} \Delta T_{\mathrm{ph}}=\Delta n_{1 \mathrm{~s}}=v_{0}\left(1-\Delta U / c_{0}^{2}\right)\left(T_{0} \Delta U / c_{0}^{2}\right) /\left(1-\Delta U / c_{0}^{2}\right)=v_{0} T_{0} \Delta U / c_{0}^{2}=\Delta U / c_{0}^{2}
$$

is the variation of $n_{1 \mathrm{~s}}$ emitted in1 s by a $h$-clock; now, see (31), $\Delta U=M_{\mathrm{E}} G h / r_{h} r_{0}$ and since $r_{h} \cong 26,600 \mathrm{~km}, r_{0} \cong 6400 \mathrm{~km}$, with $h \cong 20,200 \mathrm{~km}$, the increase of counted time in one day $\left(\Delta T_{1 d}\right)$ of a $h$-clock, with respect to a g-clock, becomes

$$
\Delta T_{1 d}=\Delta n_{1 s} \times 86400 \mathrm{~s}=86400 \Delta U / c_{0}^{2}=45.5 \mu \mathrm{s} .
$$

Now, being $v=2\left(2 \pi r_{h l} / 86,400=3870 \mathrm{~m} / \mathrm{s}\right.$ the orbital speed of GPS satellites, (corresponding to two orbits/day), it turns out that the variation of the counted time, between a $h$-clock and an Observer $E$ at the centre of Earth, due to their relative motion, is given by Equation (20) which, in our case, with $\beta=v / c$, becomes

$$
T_{E}=T_{h} \sqrt{1+\beta^{2}} \cong T_{h}\left(1+\beta^{2} / 2\right) \quad(\text { valid for } v \ll C)
$$

with $T_{E}$ the photon counted time for the Observer E; then, with $v_{0}$ the Earth's rotational speed, and since $\left(v_{0} / c\right)^{2} \ll(v / c)^{2}$, we can write $T_{0} \cong T_{E}$ with $T_{0}$ the counted time of one photon for a g-clock; so, the difference between the two transit times $T_{h}$ and $T_{0} \cong$ $T_{E}$ given by (41), is

$$
\Delta T_{\mathrm{ph}}^{\prime}=T_{h}-T_{E} \cong T_{h}-T_{0}=-T_{h} \beta^{2} / 2
$$

Then, as above, $v_{h} \Delta T_{\mathrm{ph}}^{\prime} \equiv \Delta n_{1 \mathrm{~s}}^{\prime}=-v_{h} T_{h} \beta^{2} / 2=-\beta^{2} / 2$ is the variation of the number of photons emitted by a $h$-clock in $1 \mathrm{~s}$; so the variation of the counted time, in one day, becomes

$$
\Delta T_{1 d}^{\prime}=-\left(\beta^{2} / 2\right) 86400 \mathrm{~s}=-7.2 \mu \mathrm{s}
$$

showing a decrease of the counted time for a g-clock due to the clocks relative motion; hence

$$
\Delta T_{\text {tot1d }}=\Delta T_{1 d}+\Delta T_{1 d}^{\prime}=38.3 \mu \mathrm{s} / \text { day }
$$

which is also predicted, (with different reason), by GR. To prevent the two said effects, before launching, the daily counted time ( $\left.T_{1 \mathrm{~d}}\right)$ of clocks, has to be decreased by $\cong 38 \mu \mathrm{s}$; 
this adjustment is sufficient to obtain synchronization between $h$-clocks and g-clocks: indeed, on our bases, the frequency of photons, emitted by a $h$-clock, does not change along the straight path satellite-ground, whereas as for the Relativity, because of its predicted increase of the frequency along the straight path satellite-ground, a g-clock should go, (for this $3^{\text {rd }}$ effect), out of synchronization.

\section{Gravitational redshif}

Referring to our previous paper [1], we summarize, hereafter, the differences between the Relativity and our results: as for the Relativity, the only way to explain high cosmological redshifts is the Doppler effect, (which implies an incredible universe expansion at a speed $v_{\mathrm{u}} \cong c$ ), whereas, on our results, disregarding the reciprocal motion between a (far) source and an Observer on Earth, which implies $v=v_{0}$, we get $c / \lambda=c_{0} / \lambda_{0}$, where $v_{0}, c_{0}$ and $\lambda_{0}$ are the values on Earth, showing that for $c_{0}>c$ it has to be $\lambda_{0}>\lambda$, that is a red shift. In general, the shifts observed on Earth can be therefore expressed as

$$
z \equiv \Delta \lambda / \lambda=\Delta c / c=\left(c_{0}-c\right) / c=\left(c_{0} / c\right)-1=\sqrt{U_{0} / U}-1
$$

with $U_{0}$ the potential on Earth, $U$ the one on the source. Thus, apart from Doppler effects, $z$ turns out to be the variation of $c$ (as well as $\lambda$ ) during the path of light toward a different potential. For $s<\cong 45 \mathrm{Mpc}$, [5], (corresponding to $-0.01<z<\cong 0.01$ ) if $U$ (potential on the source) is, in absolute value, higher than the potential on Earth $U_{0}$, the (45) gives, on Earth, $z<0$ (blue shift), and vice versa for $|U|<\left|U_{0}\right|$; thus, for $s<\cong 45$ $\mathrm{Mpc}$, these red/blue shifts indicate that the potential, may increase or decrease. In the range $\cong 0.01<z<\cong 0.20$, (where $z$ follows the Hubble's law), the (45), written as

$$
U=U_{0} /(1+z)^{2} \cong U_{0} /(1+2 z) \cong U_{0}(1-2 z) \quad(\text { valid for } z \ll 1)
$$

shows that, for $z \ll 1, U$ depends linearly on $z$, as Hubble's law; then, for $s \rightarrow \infty, U \rightarrow 0$, hence $z \rightarrow \infty$, see Table 1 .

Table 1. Calculated values of $U$ and $c$ related to the observed shifts on Earth.

\begin{tabular}{cccccc}
\hline blue/redshift & $\mathrm{z}$ & $s(\mathrm{Mpc})$ & $U / U_{0}=1 /(z+1)^{2}$ & $U / U_{0} \cong 1-2 z$ & $c / c_{0}=1 /(z+1)$ \\
\hline blue/red shift & $-0.01 \rightarrow 0.01$ & $<\cong 45$ & $0.98-1.02$ & $0.98-1.02$ & $0.99-1.01$ \\
red shift & $\cong 0.01$ & $\cong 45$ & 0.98 & 0.98 & 0.99 \\
red shift & 0.20 & 900 & 0.69 & 0.60 & 0.83 \\
red shift & 1 & & 0.25 & & 0.50 \\
red shift & $\rightarrow \infty$ & $\rightarrow \infty$ & $\rightarrow 0$ & & $\rightarrow 0$ \\
\hline
\end{tabular}

For $s>\cong 45 \mathrm{Mpc}, z$ is always positive, hence we may argue that our galaxy is close to the centre of potential $C_{p}$ of all the masses, (where $\left|U_{\mathrm{Cp}}\right|$ has the max value), practically close to the middle of the masses of universe.

\section{Part 2-Interaction Light-Matter}

\subsection{Electron Structure and Photon-Electron Impact Point}

On our basis, (light composed of our photons), the interaction light-matter requires 
that to move a circling electron toward outer orbits, the impact photon-electron has to occur, see Figure 7(a), in a radial way, (giving origin to the radial velocity w), otherwise, some impacts could cause the electron fall into the nucleus, due, for instance, to an impact where photons-electron have contrary direction.

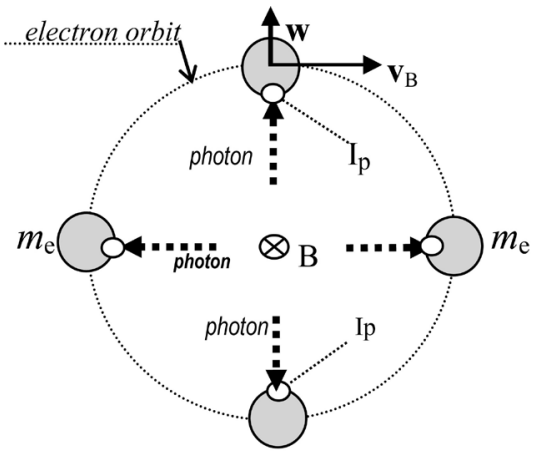

(a) - Electron orbit front view;

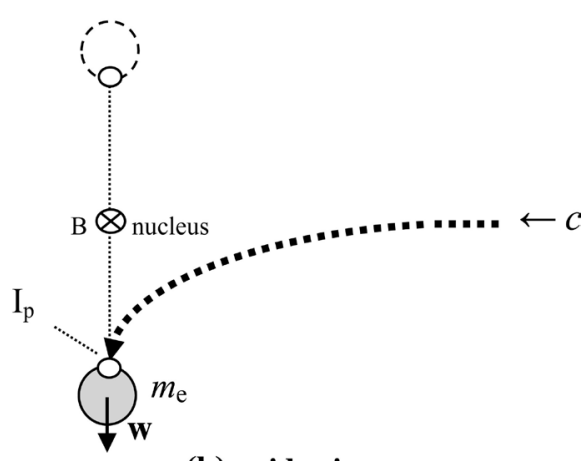

(b) - side view

Figure 7. Photon-electron Impact point $\left(\mathrm{I}_{\mathrm{p}}\right)$ and electron radial velocity w.

To be radial, the impact must occur in a specific point (Impact Point, fixed to the electron) which, during the electron revolution, has to face its nucleus, (up to its removal), giving to the electron one rotation every revolution. Thus we can infer that the electric charge of the electron, has to correspond to the Impact Point $\left(\mathrm{I}_{\mathrm{p}}\right)$, and we have also to argue that each photon front is provided with a positive charge, while its tail with an equal negative one.

Moreover, in case of more impacts, as it happens, for instance, on Absorption/ Emission effect, where the impacts move a circling electron toward higher orbits, the impacts photons-electron have to occur all around the electron orbit, thus the impacting photons have to approach the nucleus, as shown on Figure $7(\mathrm{~b})$, perpendicularly to the electron orbit plane, providing, to the electron, a radial velocity $\mathbf{w}$.

\subsection{H Atom Parameters (On Our Bases) and Meaning of Its Quantum Numbers}

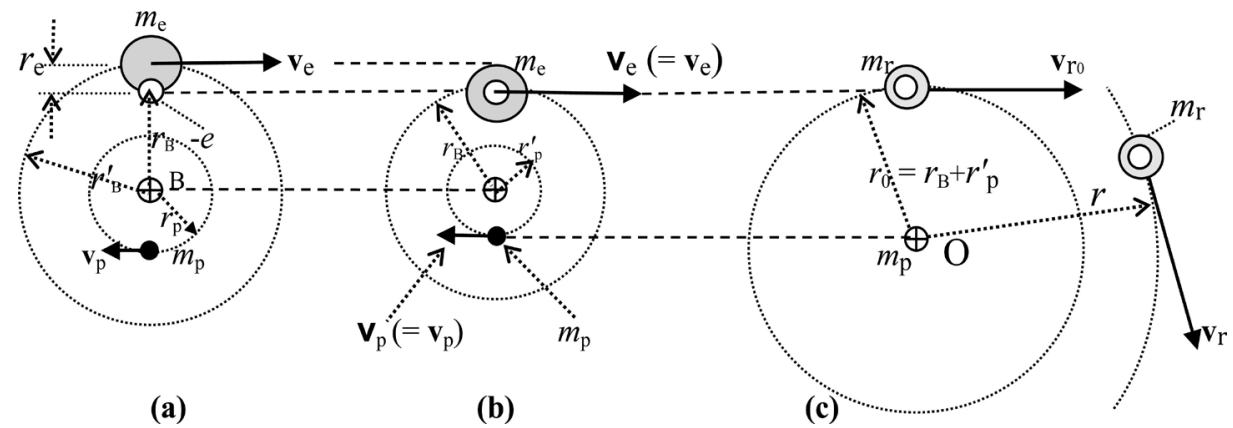

Figure 8. H atom configurations, (on our bases), at constant total energy. (a) Observed from the electron-proton common centre of gravity B; (b) Ditto, with the electron barycentre now coincident with its proper charge; (c) Observed from the proton fixed as origin, orbited by the electron having now a reduced mass. 
The Figure 8(a) represents an $\mathrm{H}$ atom with both the electron mass $m_{\mathrm{e}}$ and the proton mass $m_{\mathrm{p}}$ circling around $\mathrm{B}$ (centre of mass of the system electron-proton), and where, on our bases:

$r_{\mathrm{B}}$ is the ground-state orbit of the electron charge,

$r_{\mathrm{e}}$ the electron radius,

$r_{\mathrm{p}}$ the proton ground-state orbit,

$V_{\mathrm{e}}=\left|\mathbf{v}_{\mathrm{e}}\right|$, the electron ground-state (orbital) speed,

$V_{\mathrm{p}}$ the proton ground-state speed.

Hence, the ground-state orbit of the electron barycentre turns out to be

$$
r_{\mathrm{B}}^{\prime}=r_{\mathrm{B}}+r_{\mathrm{e}}=r_{\mathrm{B}}\left(1+r_{\mathrm{e}} / r_{\mathrm{B}}\right)=r_{\mathrm{B}}\left(1+\varepsilon_{\mathrm{r}}\right),\left(\text { with } \varepsilon_{\mathrm{r}} \equiv r_{\mathrm{e}} / r_{\mathrm{B}}\right) .
$$

Now, to apply properly the equality between the electron centrifugal force and the Coulomb force, we have to consider the configuration (c) where the electron reduced mass

$$
m_{\mathrm{r}}=m_{\mathrm{e}} /\left(1+m_{\mathrm{e}} / m_{\mathrm{p}}\right)=m_{\mathrm{e}} /\left(1+\varepsilon_{\mathrm{m}}\right) \quad\left(\text { with } \quad \varepsilon_{\mathrm{m}}=m_{\mathrm{e}} / m_{\mathrm{p}}\right),
$$

is circling around the proton fixed as origin $(\mathrm{O})$. Now, the total energy of the configurations $(\mathbf{a})$ and $(\mathbf{b})$ are:

Configuration (a), where $m_{\mathrm{e}} v_{\mathrm{e}}=m_{\mathrm{p}} v_{\mathrm{p}} \Rightarrow v_{\mathrm{p}}=m_{\mathrm{e}} v_{\mathrm{e}} / m_{\mathrm{p}}=\varepsilon_{\mathrm{m}} v_{\mathrm{e}}$;

$$
T_{\mathrm{a}}=m_{\mathrm{e}} v_{\mathrm{e}}^{2}+m_{\mathrm{p}} v_{\mathrm{p}}^{2}=m_{\mathrm{e}} v_{\mathrm{e}}^{2}\left(1+m_{\mathrm{e}} / m_{\mathrm{p}}\right)=m_{\mathrm{e}} v_{\mathrm{e}}^{2}\left(1+\varepsilon_{\mathrm{m}}\right)
$$

Configuration (b), where $m_{\mathrm{e}} v_{\mathrm{e}}=m_{\mathrm{p}} v_{\mathrm{p}} \Rightarrow v_{\mathrm{p}}=m_{\mathrm{e}} v_{\mathrm{e}} / m_{\mathrm{p}}=\varepsilon_{\mathrm{m}} v_{\mathrm{e}}$;

$$
T_{\mathrm{b}}=m_{\mathrm{e}} V_{\mathrm{e}}^{2}+m_{\mathrm{p}} V_{\mathrm{p}}^{2}=m_{\mathrm{e}} V_{\mathrm{e}}^{2}\left(1+m_{\mathrm{e}} / m_{\mathrm{p}}\right)=m_{\mathrm{e}} V_{\mathrm{e}}^{2}\left(1+\varepsilon_{\mathrm{m}}\right)
$$

hence for $T_{\mathrm{a}}=T_{\mathrm{b}}$ we get $v_{\mathrm{e}}=V_{e}$ and $v_{\mathrm{p}}=V_{\mathrm{p}}$. (As for $T_{c}=\left(T_{\mathrm{a}}=T_{b}\right)$, next chapter).

Now, see Figure 8(b), $r_{\mathrm{p}}^{\prime}$ can be found from the relation $v_{\mathrm{e}} / r_{\mathrm{B}}=v_{\mathrm{p}} / r_{\mathrm{p}}^{\prime}$ yielding

$$
r_{\mathrm{p}}^{\prime}=v_{\mathrm{p}} r_{\mathrm{B}} / v_{\mathrm{e}}=\left(\frac{v_{\mathrm{e}} m_{\mathrm{e}}}{m_{\mathrm{p}}}\right) r_{\mathrm{B}} / v_{\mathrm{e}}=r_{\mathrm{B}} m_{\mathrm{e}} / m_{\mathrm{p}}=r_{\mathrm{B}} \varepsilon_{\mathrm{m}}
$$

thus

$$
r_{0}=r_{\mathrm{B}}+r_{\mathrm{p}}^{\prime}=r_{\mathrm{B}}+r_{\mathrm{B}} \varepsilon_{\mathrm{m}}=r_{\mathrm{B}}\left(1+\varepsilon_{\mathrm{m}}\right)=r_{\mathrm{B}}^{\prime}\left(1+\varepsilon_{\mathrm{m}}\right) /\left(1+\varepsilon_{\mathrm{r}}\right)
$$

Now, see Figure 8(c), equating along a circular orbit, the electron centrifugal force to the Coulomb one

$$
m_{\mathrm{r}} v_{\mathrm{r}}^{2}=e^{2} / 4 \pi \varepsilon_{0} r,
$$

and calling $U_{\mathrm{r} \infty}=\int_{r}^{\infty}\left(e^{2} / 4 \pi \varepsilon_{0} r^{2}\right) \mathrm{d} r$ the necessary and sufficient energy to move the electron charge from $r$ toward $\infty$, and assuming $U_{\infty}=0$, we get

$$
U_{\mathrm{r} \infty}\left(=-U_{\mathrm{r}}\right)=e^{2} / 4 \pi \varepsilon_{0} r
$$

where $U_{\mathrm{r}}=-e^{2} / 4 \pi \varepsilon_{0} r$ is the potential due to electrostatic attraction. So the (51) becomes

$$
m_{\mathrm{r}} v_{\mathrm{r}}^{2}=U_{\mathrm{ro}}\left(=-U_{\mathrm{r}}\right)
$$

Now, the orbital kinetic energy of $m_{\mathrm{r}}$ is $K_{\mathrm{e}}=\frac{1}{2} m_{\mathrm{r}} v_{\mathrm{r}}^{2}$, thus, with $W$ the related ioni- 
zation energy, (that is the electron extraction work), we may write

$$
U_{\mathrm{r} \infty}=m_{\mathrm{r}} v_{\mathrm{r}}^{2}=\frac{1}{2} m_{\mathrm{r}} v_{\mathrm{r}}^{2}+\frac{1}{2} m_{\mathrm{r}} v_{\mathrm{r}}^{2} \Rightarrow W\left(=K_{\mathrm{e}}\right)=\frac{1}{2} m_{\mathrm{r}} v_{\mathrm{r}}^{2}
$$

The term $E=h v$, see (21), is the energy of light passing along one ray, thus, if the ionization energy $W\left(=\frac{1}{2} m_{\mathrm{r}} v_{\mathrm{r}}^{2}\right)$ is supplied by one ray of light (with energy $E=h v$ ) it must be

$$
W(=E) \Rightarrow \frac{1}{2} m_{\mathrm{r}} v_{\mathrm{r}}^{2}=h v
$$

Therefore, substituting $2 h v\left(=m_{\mathrm{r}} v_{\mathrm{r}}^{2}\right)$ into (51) and solving by $r$ we get

$$
r=e^{2} / 4 \pi \varepsilon_{0} 2 h v=e^{2} / 4 \pi \varepsilon_{0} m_{\mathrm{r}} v_{\mathrm{r}}^{2}
$$

Now, plugging into (56) the value of the highest $\mathrm{H}$-atom spectrum frequency $v_{0}=c / \lambda_{0}$ $=c R_{\mathrm{H}}$ where $R_{\mathrm{H}}\left(=1 / \lambda_{0}\right)$ is the Rydberg constant, whose experimental value is $R_{\mathrm{H}}=$ $10,967,758 \mathrm{~m}^{-1}$, we get, as for $\mathrm{H}$ atom, see Figure $8(\mathrm{c})$, the ground-state orbit (referred to the proton) of the reduced electron

$$
r_{0}=e^{2} / 8 \pi \varepsilon_{0} h v_{0}=e^{2} / 8 \pi \varepsilon_{0} h R_{\mathrm{H}} c=\alpha / 4 \pi R_{\mathrm{H}}=5.294654 \times 10^{-11} \mathrm{~m}
$$

with $\alpha=e^{2} / 2 \varepsilon_{\mathrm{o}} h c$ the fine structure constant. Then writing the (50) as $r_{\mathrm{B}}=r_{0} /\left(1+\varepsilon_{\mathrm{m}}\right)$, we get

$$
r_{\mathrm{B}}=r_{0} /\left(1+m_{\mathrm{e}} / m_{\mathrm{p}}\right)=e^{2} / 8 \pi \varepsilon_{0} h R_{\infty} c=\alpha / 4 \pi R_{\infty}=5.291772 \times 10^{-11} \mathrm{~m}
$$

corresponding to the Bohr radius, with $R_{\infty}=R_{\mathrm{H}}\left(1+m_{\mathrm{e}} / m_{\mathrm{p}}\right)$, whereas, on our bases, $r_{\mathrm{B}}$ corresponds, see Figure $8(\mathrm{~b})$, to the ground-state orbit of the electron around the electron-proton centre of mass B.

Now, the speed of $m_{\mathrm{r}}$ along the orbit $r_{0}$, from (55), becomes

$$
v_{\mathrm{r} 0}=\sqrt{2 h v_{0} / m_{\mathrm{r}}}=\sqrt{2 h R_{\mathrm{H}} c / m_{\mathrm{r}}}=2187691.2 \mathrm{~m} \cdot \mathrm{s}^{-1}
$$

and given the frequency of the electron $m_{\mathrm{r}}$ along $r_{0}$ that is $v_{\mathrm{e} 0}=V_{\mathrm{r} 0} / 2 \pi r_{0}$, the ratio $2 v_{0} / v_{\mathrm{e} 0}$, with $r_{0}\left(=\alpha / 4 \pi R_{\mathrm{H}}\right)$ as given by (57), becomes

$$
\begin{aligned}
2 v_{0} / v_{\mathrm{e} 0} & =2 c R_{\mathrm{H}} / v_{\mathrm{e} 0}=2 c R_{\mathrm{H}} /\left(v_{\mathrm{r} 0} / 2 \pi r_{0}\right)=2 c R_{\mathrm{H}} / \sqrt{\left(2 h R_{\mathrm{H}} c / m_{\mathrm{r}}\right)} / 2 \pi r_{0} \\
& =2 c R_{\mathrm{H}} / \sqrt{\left(2 h R_{\mathrm{H}} c / m_{\mathrm{r}}\right)} /\left(2 \pi \alpha / 4 \pi R_{\mathrm{H}}\right)=\alpha c / \sqrt{2 h R_{\mathrm{H}} c / m_{\mathrm{r}}}=1.00000001
\end{aligned}
$$

hence it is consistent to assume $2 v_{0} / v_{\mathrm{e} 0}=1$ (exactly). This ratio, written $2 T_{\mathrm{e} 0}=T_{0}$, implies that, on $\mathrm{H}$ atom, the light-electron impact time $T_{0}$ lasts for two electron orbits.

Now, it is known that the admitted wavelengths, along circular orbits, have to satisfy the relation $\lambda_{n}=n^{2} \lambda_{0}$, with $n=1,2,3, \cdots$ an integer, so we can also write

$$
v_{n}=v_{0} / n^{2}(n=1,2,3, \cdots)
$$

with $v_{n}$ the photons admitted frequency along circular orbits. Then from (56) we can write

$$
r_{n}=e^{2} / 4 \pi \varepsilon_{0} 2 h v_{n}=e^{2} n^{2} / 4 \pi \varepsilon_{0} 2 h v_{0}=r_{0} n^{2}
$$

representing the radius of each circular orbit. Then, see (59), the orbital speed of the electron $m_{\mathrm{r}}$ along any circular orbit is

$$
v_{n}=\sqrt{2 h v_{n} / m_{\mathrm{r}}}=\sqrt{2 h v_{0} / n^{2} m_{\mathrm{r}}}=v_{\mathrm{r} 0} / n
$$


while its frequency is

$$
v_{\mathrm{en}}=v_{n} / 2 \pi r_{n}=v_{\mathrm{r} 0} / n 2 \pi r_{0} n^{2}=v_{\mathrm{e} 0} / n^{3} .
$$

Then, dividing (61) by (64) and because of the ratio $2 v_{\mathrm{o}} / v_{\mathrm{eo}}=1$, we get

$$
v_{n} / v_{\mathrm{en}}=\left(v_{0} / v_{\mathrm{e} 0}\right) n \Rightarrow 2 v_{n} / v_{\mathrm{e} n}=n(n=1,2,3, \cdots)
$$

Now, as $v(=n / t)$ is the number $n$ of photons passing along one ray during $t$, for $t=$ $2 T_{\mathrm{e}}$ one gets $v=n / 2 T_{\mathrm{e}}=n v_{\mathrm{e}} / 2$, which equals the (65), so the integer $n$ of (65) also represents the number of photons (of the same ray) absorbed (or emitted) by the electron during $2 T_{\mathrm{e}}$ and this number, for all the $n$ circular orbits of $H$ atom, is an integer starting with 1 along the two orbits related to the photon $v_{0}$. [Between two circular orbits, the photons frequency are shown on Section 3.5].

The (65) written as $n T_{n}=2 T_{\text {en }}$ shows that the impact time $n T_{n}$ of $n$ photons (with frequency $v_{n}$ ) equals the time needed by the electron, along the orbit $r_{n}$, for two orbits.

For $n=1$, the Equation (65) corresponds to $2 v_{0} 2 \pi r_{0} / v_{\mathrm{r} 0}=1$ and substituting here $r_{0}$ $\left(=e^{2} / 8 \pi \varepsilon_{0} h v_{0}\right)$, as given by (57), we get $4 \pi v_{0} e^{2} / 8 \pi \varepsilon_{0} h v_{0} v_{\mathrm{r} 0}=1$, that is

$$
4 \pi v_{0} e^{2} / 8 \pi \varepsilon_{0} h v_{0}=v_{\mathrm{r} 0} \Rightarrow v_{\mathrm{r} 0}=e^{2} / 2 \varepsilon_{0} h=\alpha c=2187691.2 \mathrm{~m} \cdot \mathrm{s}^{-1}
$$

same value given by (59). Now, comparing (59) to (66) we find

$$
\sqrt{2 h R_{\mathrm{H}} c / m_{\mathrm{r}}}=\alpha c \Rightarrow R_{\mathrm{H}}=\alpha^{2} c^{2} m_{\mathrm{r}} / 2 h c=10967758 \mathrm{~m}^{-1}
$$

matching the $R_{\mathrm{H}}$ experimental value.

\subsection{Impact Photon-Electron and Electron Radial Speed}

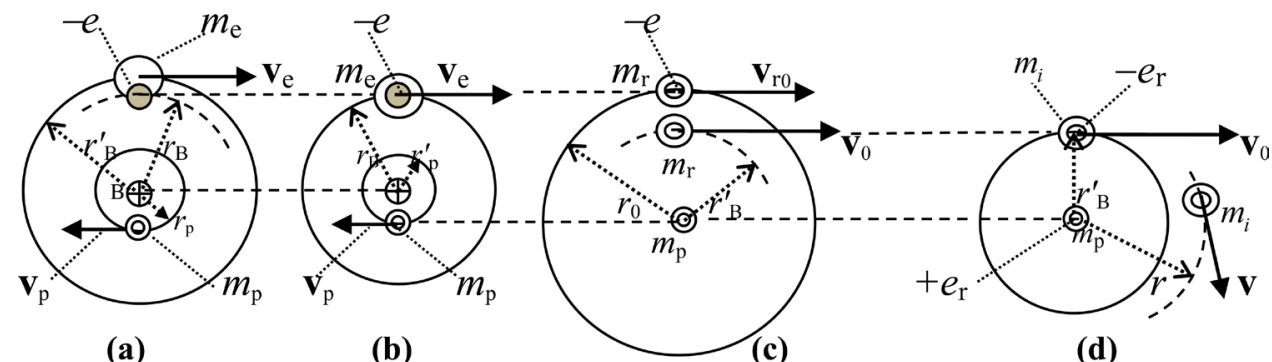

Figure 9. H atom configurations. (a) observed from the common centre of gravity B. (b) ditto, with the electron centre now coincident with its proper charge. (c) observed from the nucleus fixed as origin, with the reduced electron mass $m_{\mathrm{r}^{*}}$ (d) observed from the nucleus, with $m_{\mathrm{i}}$ (here called electron impact mass) circling along the effective orbit $r_{\mathrm{B}}^{\prime}$, with two reduced charges $\left(-e_{\mathrm{r}}\right.$, $\left.+e_{\mathrm{r}}\right)$.

Still referring to $\mathrm{H}$ atom, to apply properly the Conservation of momentum (CoM) to the impact photon-electron, we need, see Figure 9, a proper configuration where its nucleus should be fixed in the common centre of gravity B orbited by an equivalent electron mass $m$, as shown on Figure $9(\mathrm{~d})$.

The Figure 9 shows the necessary passages, from Figures $9(a)-(d)$, to obtain such a configuration.

Referring to Figure $9(\mathrm{c})$, with $m_{\mathrm{r}}$ circling around $r_{\mathrm{B}}^{\prime}$, with orbital speed $v_{0}$, the (51) 
gives

$$
m_{\mathrm{r}} v_{0}^{2} r_{\mathrm{B}}^{\prime}=e^{2} / 4 \pi \varepsilon_{0}
$$

and substituting, see $(50), \quad r_{\mathrm{B}}^{\prime}=r_{0}\left(1+\varepsilon_{\mathrm{r}}\right) /\left(1+\varepsilon_{\mathrm{m}}\right)$, we can write

$$
m_{\mathrm{r}} v_{0}^{2} r_{0}\left(1+\varepsilon_{\mathrm{r}}\right) /\left(1+\varepsilon_{\mathrm{m}}\right)=e^{2} / 4 \pi \varepsilon_{0}
$$

then calling

$$
m_{\mathrm{i}} \equiv m_{\mathrm{r}}\left(1+\varepsilon_{\mathrm{r}}\right) /\left(1+\varepsilon_{\mathrm{m}}\right)
$$

electron impact mass, we get

$$
m_{\mathrm{i}} v_{0}^{2} r_{0}=e^{2} / 4 \pi \varepsilon_{0}
$$

and therefore

$$
m_{\mathrm{i}} v_{0}^{2} r_{\mathrm{B}}^{\prime}=\left[\left(1+\varepsilon_{\mathrm{r}}\right) /\left(1+\varepsilon_{\mathrm{m}}\right)\right] e^{2} / 4 \pi \varepsilon_{0}
$$

we can now write

$$
m_{\mathrm{i}} v_{0}^{2} r^{\prime}=e_{\mathrm{r}}^{2} / 4 \pi \varepsilon_{0} \text { where } e_{\mathrm{r}}=e \sqrt{\left(1+\varepsilon_{\mathrm{r}}\right) /\left(1+\varepsilon_{\mathrm{m}}\right)}
$$

showing, see Figure 9(d), $m$ circling along the orbit $r_{\mathrm{B}}^{\prime}$, implying an electron/proton reduced charge $e_{\mathrm{r}}$. Now, referring to Figure $9(\mathrm{c})$, we have $m_{\mathrm{r}} v_{\mathrm{r} 0}^{2} r_{0}=m_{\mathrm{r}} v_{0}^{2} r_{\mathrm{B}}^{\prime}$, and since, see (66), $v_{\mathrm{r} 0}=\alpha c$, the orbital speed of $m_{\mathrm{r}}$ along the orbit $r_{\mathrm{B}}^{\prime}$ becomes

$$
v_{0}=v_{\mathrm{r} 0} \sqrt{\left(1+\varepsilon_{\mathrm{m}}\right) /\left(1+\varepsilon_{\mathrm{r}}\right)}=\alpha c \sqrt{\left(1+\varepsilon_{\mathrm{m}}\right) /\left(1+\varepsilon_{\mathrm{r}}\right)}
$$

which, given $\varepsilon_{\mathrm{m}}$ and $\varepsilon_{\mathrm{r}}$, leads to $v_{0}=c / 137$, as shown on next chapter.

We compare now the total energy $T$ of the system electron-proton along the configurations of Figure 9: as for Figure 9(a) and Figure 9(b), the total energy of the system is $T_{\mathrm{a}}=T_{\mathrm{b}}=m_{\mathrm{e}} v_{\mathrm{e}}^{2}\left(1+\varepsilon_{\mathrm{m}}\right)$; as for Figure $9(\mathrm{c})$, along $r_{0}$, we get $T_{\mathrm{c}}=m_{\mathrm{r}} v_{\mathrm{r} 0}^{2}=m_{\mathrm{r}}\left(v_{\mathrm{e}}+v_{\mathrm{p}}\right)^{2}=m_{\mathrm{r}}\left[v_{\mathrm{e}}\left(1+\varepsilon_{\mathrm{m}}\right)\right]^{2}=\left[m_{\mathrm{e}} /\left(1+\varepsilon_{\mathrm{m}}\right)\right] v_{\mathrm{e}}^{2}\left(1+\varepsilon_{\mathrm{m}}\right)^{2}=m_{\mathrm{e}} v_{\mathrm{e}}^{2}\left(1+\varepsilon_{\mathrm{m}}\right)=T_{\mathrm{a}}$ ; on $6(\mathrm{~d}), \quad T_{\mathrm{d}}=m_{\mathrm{i}} v_{0}^{2}=\left[m_{\mathrm{r}}\left(1+\varepsilon_{\mathrm{r}}\right) /\left(1+\varepsilon_{\mathrm{m}}\right)\right] v_{\mathrm{r} 0}^{2}\left[\left(1+\varepsilon_{\mathrm{m}}\right) /\left(1+\varepsilon_{\mathrm{r}}\right)\right]=m_{\mathrm{r}} v_{\mathrm{r} 0}^{2}=T_{\mathrm{c}}$.

The conservation of momentum, applied before and after an inelastic impact photon-electron, since photon and w, see Figure 7, have same direction, the (29), for a generic atom, gives

$$
w=2 m c / m_{\mathrm{e}}=2 \gamma v c / m_{\mathrm{e}}=\left(2 \gamma c / m_{\mathrm{e}}\right) / T=2 \gamma v c^{2} / \mathrm{cm}_{\mathrm{e}}=2 h v / \mathrm{cm} \mathrm{e}_{\mathrm{e}}
$$

representing the electron radial speed originated by an impact of one photon during the impact time $T$, while for $n$ photons (with frequency $v$ ) we have

$$
w_{n}=n w=n 2 m c / m_{\mathrm{e}}=2 n \gamma v c / m_{\mathrm{e}}=2 n h v / c m_{\mathrm{e}} .
$$

Regarding now the $\mathrm{H}$ atom and referring to Figure 9(c), meaning to consider the electron reduced mass $m_{\mathrm{r}}$ circling along $r_{0}$, the (75) for $v=v_{0}$, becomes

$$
w_{0}=\frac{2 h v_{0}}{c m_{\mathrm{r}}}=\sqrt{2 h v_{0} / m_{\mathrm{r}}} \sqrt{2 h v_{0} / m_{\mathrm{r}}} / c
$$

and since, see (59), $\quad v_{\mathrm{r} 0}=\left(2 h v_{0} / m_{\mathrm{r}}\right)^{1 / 2}=\alpha c$, as shown by (66), we get

$$
w_{0}=v_{\mathrm{r} 0}^{2} / c=\alpha^{2} c=15964.35 \mathrm{~m} \cdot \mathrm{s}^{-1}
$$


while considering the configuration $9(\mathrm{~d})$, where the electron $m_{\mathrm{i}}$ is circling along $r_{\mathrm{B}}^{\prime}$, we get

$w_{0}=2 h v_{0} / c m=\alpha^{2} c\left(1+\varepsilon_{\mathrm{m}}\right) /\left(1+\varepsilon_{\mathrm{r}}\right)=15,973.048 \mathrm{~m} \cdot \mathrm{s}^{-1} \quad\left(\right.$ valid for $\left.\quad \varepsilon_{\mathrm{r}}=r_{\mathrm{e}} / r_{\mathrm{B}}=0\right)$

close to $c / 137^{2}=15,972.743 \mathrm{~m} \mathrm{~s}^{-1}$; on next chapter, via $r_{\mathrm{e}}$, we get $w_{0}=c / 137^{2}$ and $v_{0}=$ c/137.

\subsection{Ionization Condition, Number of Electron Circular Orbits (H Atom), and Electron Radii}

Now, referring to Figure 10, let us consider an electron $\left(m_{\mathrm{e}}\right)$ circling, with velocity $\mathbf{v}$ around its nucleus with mass $m_{\mathrm{N}} \gg m_{\mathrm{e}}$, which can therefore be considered as fixed in the atom centre of gravity B; its removal may happen when its radial speed $w$ equals $V$ $(=|\mathbf{v}|)$ that is

$$
w=v(\text { ionization condition }) \text {. }
$$

In particular, as for $\mathrm{H}$ atom, and referring to Figure $9(\mathrm{c})$, let us consider the electron $m_{\mathrm{r}}$ circling along $r_{0}$; comparing (78), that is $w_{0}=\alpha^{2} c$, with (66) that is $V_{\mathrm{r} 0}=\alpha c$, we get $w_{0} / v_{\mathrm{r} 0}=\alpha \cong 1 / 137 \ll 1$.

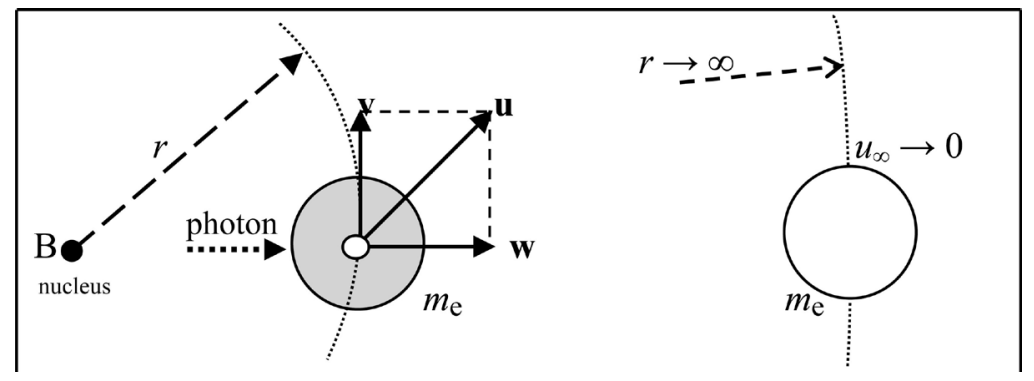

Figure 10. Ionization condition $(w=v)$.

Now, along $r_{0}$, (ground-state orbit), we have $n=1$ (meaning one photon along the double orbit $r_{0}$ ), hence the ionization, requiring $w_{0} / V_{\mathrm{r} 0}=1$, cannot happen along $r_{0}$. Referring now to Figure 9(d), along the ionization double orbit (in short d-orbit) \# $n_{\mathrm{i}}$, where the photons incident frequency, see (61), is $v_{\mathrm{i}}=v_{0} / n_{\mathrm{i}}^{2}$, the impact due to $n_{\mathrm{i} \text { - }}$ photons would produce, considering the electron impact mass $m_{\mathrm{i}}$, an electron radial speed, see (76), equal to

$$
w_{n \mathrm{i}}=2 n_{\mathrm{i}} h v_{0} / n_{\mathrm{i}}^{2} c m_{\mathrm{i}}=2 h v_{0} / n_{\mathrm{i}} c m_{\mathrm{r}}\left(1+\varepsilon_{\mathrm{r}}\right) /\left(1+\varepsilon_{\mathrm{m}}\right)=w_{0} / n_{\mathrm{i}}\left(1+\varepsilon_{\mathrm{r}}\right) /\left(1+\varepsilon_{\mathrm{m}}\right) \cong w_{0} / n_{\mathrm{i}}
$$

and since along this orbit $\left(n_{\mathrm{i}}\right)$, see (63), is $v_{\mathrm{i}}=v_{0} / n_{\mathrm{i}}$, where $v_{0}$ is given by Equation (74), we find

$$
w_{n i} / v_{\mathrm{i}} \cong\left(w_{0} / n_{\mathrm{i}}\right) /\left(v_{0} / n_{\mathrm{i}}\right)=w_{0} / v_{0}=\alpha \cong 1 / 137 \ll 1
$$

hence the ionization, which requires $w_{n i}=V_{\mathrm{i}}$, with only $n_{\mathrm{i}}$ photons along the $n_{\mathrm{i}}^{\text {th }} \mathrm{d}$-orbit, would never happen; thus we must infer that there are 137 progressive $\mathrm{d}$-orbits, where the electron is circling $n$ times along every d-orbit; thus the number of photons admitted along $n$ d-orbits turns out to be $n^{2}$, yielding to the radial speed, along $n$ ionization 
d-orbits $n_{\mathrm{i}}$, the value $w_{n^{2}}=n_{\mathrm{i}} w_{n \mathrm{i}}$ which becomes

$$
w_{n^{2}}=2 n_{\mathrm{i}}^{2} h v_{0} / n_{\mathrm{i}}^{2} c m_{i}=2 h v_{0} / c m_{i}=w_{0}
$$

giving the same radial speed $w_{0}$ for any circular d-orbit, see Table 2 .

Table 2. Ionization parameters of $\mathrm{H}$ atom (on the last column, $3^{\text {rd }}$ line, change $w_{n^{2}}$ according to the new value $2 n^{2} \gamma c v_{0} / n^{2} m_{i}$ ).

\begin{tabular}{|c|c|c|c|c|c|}
\hline $\begin{array}{c}\text { Progressive number of } \\
\text { each circular (double) } \\
\text { orbit. } \\
n^{\text {th }}\end{array}$ & $\begin{array}{l}\text { Number of photons } \\
\text { along each (double) } \\
\text { orbit. } \\
n\end{array}$ & $\begin{array}{l}\text { Number of photons } \\
\text { along } n \text { (double) } \\
\text { orbits. } \\
n^{2} \\
-\end{array}$ & $\begin{array}{c}\text { Photons frequency } \\
\text { along the } n \text {th (double) } \\
\text { orbit. } \\
v_{n}=v_{0} / n^{2} \\
\left(\times 10^{10} \mathrm{~Hz}\right)\end{array}$ & $\begin{array}{l}\text { Electron orbital } \\
\text { speed along the } n^{\text {th }} \\
\text { orbit. } \\
v_{n}=v_{0} / n \\
\mathrm{~m} / \mathrm{s}\end{array}$ & $\begin{array}{l}\text { Electron radial speed due to } \\
n^{2} \text { photons with frequency } v_{n} \text {. } \\
\qquad \begin{array}{c}w_{n^{2}}=2 n^{2} \gamma c v_{0} / n^{2} m_{i} \\
\mathrm{~m} / \mathrm{s}\end{array}\end{array}$ \\
\hline $1^{\mathrm{st}}$ & 1 & 1 & $328,805.1$ & $2,188,266$ & $15,972.74\left(=w_{0}\right)$ \\
\hline $137^{\text {th }}$ & 137 & $137^{2}$ & 17.52 & $15,972.74$ & $15,972.74=c / 137^{2}$ \\
\hline
\end{tabular}

Now we can obtain the radius $\left(r_{\mathrm{e}}\right)$ of the electron: indeed, along the ionization d-orbit $n_{\mathrm{i}}$, the ionization condition $w_{n^{2}}=v_{\mathrm{i}}$ becomes $w_{0}=v_{0} / n_{\mathrm{i}}$, and plugging $v_{0}$ as giving by (74) we get

$$
n_{\mathrm{i}}=v_{0} / w_{0}=\alpha c \sqrt{\left(1+\varepsilon_{\mathrm{m}}\right) /\left(1+\varepsilon_{\mathrm{r}}\right)} / w_{0}
$$

where $w_{0}$ is given by (77) and since $R_{\mathrm{H}}\left(=1 / \lambda_{0}=v_{0} / c\right)=\alpha^{2} c m_{\mathrm{r}} / 2 h$, see (67), we have

$$
\begin{aligned}
n_{\mathrm{i}} & =\alpha c \sqrt{\left(1+\varepsilon_{\mathrm{m}}\right) /\left(1+\varepsilon_{\mathrm{r}}\right)} /\left(\frac{2 h v_{0}}{c m_{\mathrm{i}}}\right)=\alpha c^{2} m_{\mathrm{i}}\left[\sqrt{\left(1+\varepsilon_{\mathrm{m}}\right) /\left(1+\varepsilon_{\mathrm{r}}\right)}\right] / 2 h v_{0} \\
& =\alpha c^{2} m_{\mathrm{r}}\left[\sqrt{\left(1+\varepsilon_{\mathrm{r}}\right)} / \sqrt{\left(1+\varepsilon_{\mathrm{m}}\right)}\right] / 2 h R_{\mathrm{H}} c \\
& =\alpha c^{2} m_{\mathrm{r}}\left[\sqrt{\left(1+\varepsilon_{\mathrm{r}}\right)} / \sqrt{\left(1+\varepsilon_{\mathrm{m}}\right)}\right] / 2 h\left(\alpha^{2} c m_{\mathrm{r}} / 2 h\right) c \\
& =(1 / \alpha)\left[\sqrt{\left(1+\varepsilon_{\mathrm{r}}\right)} / \sqrt{\left(1+\varepsilon_{\mathrm{m}}\right)}\right]=136.99869 \quad\left(\text { valid for } \varepsilon_{\mathrm{r}}=0\right)
\end{aligned}
$$

but $n_{\mathrm{i}}$ has to be an integer so we can infer $n_{\mathrm{i}}=137$, giving

$$
137^{2} \alpha^{2}\left(1+\varepsilon_{\mathrm{m}}\right)=\left(1+\varepsilon_{\mathrm{r}}\right) \Rightarrow \varepsilon_{\mathrm{r}}=137^{2} \alpha^{2}\left(1+\varepsilon_{\mathrm{m}}\right)-1
$$

yielding

$$
r_{\mathrm{e}}=r_{\mathrm{B}}\left[137^{2} \alpha^{2}\left(1+\varepsilon_{\mathrm{m}}\right)-1\right]=\left(\alpha / 4 \pi R_{\infty}\right)\left[137^{2} \alpha^{2}\left(1+m_{\mathrm{e}} / m_{\mathrm{p}}\right)-1\right]=1.005172 \mathrm{fm}
$$

Now, the correct values of $m_{\mathrm{i}}, w_{\mathrm{o}}$ and $v_{\mathrm{o}}$ from (70), (79), (74), with $\varepsilon_{\mathrm{r}}$ given by (86), become

$$
\begin{gathered}
m_{\mathrm{i}}=m_{\mathrm{r}}\left(1+\varepsilon_{\mathrm{r}}\right) /\left(1+\varepsilon_{\mathrm{m}}\right)=m_{\mathrm{r}} 137^{2} \alpha^{2}=9.0996407 \times 10^{-31} \mathrm{~kg}, \\
w_{0}=\alpha^{2} c\left(1+\varepsilon_{\mathrm{m}}\right) /\left(1+\varepsilon_{\mathrm{r}}\right)=c / 137^{2}=15972.743 \mathrm{~m} \cdot \mathrm{s}^{-1}, \\
v_{0}=\alpha c \sqrt{\left(1+\varepsilon_{\mathrm{m}}\right) /\left(1+\varepsilon_{\mathrm{r}}\right)}=c / 137=2188266.1 \mathrm{~m} \cdot \mathrm{s}^{-1} .
\end{gathered}
$$

Now, the (84), that is $n_{\mathrm{i}}=v_{0} / w_{0}$, through (89) and (90) yields

$$
n_{\mathrm{i}}=v_{0} / w_{0}=(1 / \alpha) \sqrt{\left(1+\varepsilon_{\mathrm{m}}\right) /\left(1+\varepsilon_{\mathrm{r}}\right)}=137
$$


where $n_{\mathrm{i}}$, on Absorption effect, is a specific constant representing the number of circular orbits as well as the numbers of admitted photons along theionization orbit.

\subsection{Absorption/Emission Effect: Photons Admitted Frequencies, Claimed Fall of Circling Electron}

Referring to Figure 11, where we represent the Absorption of photons from acircling electron, let us assume the nucleus mass $m_{\mathrm{N}} \gg m_{\mathrm{e}}$, so to consider the nucleus fixed in the atom centre of gravity B. Now, the expression of the total energy of the system photon-electron is given by

$$
T=E+U_{r}+K_{\mathrm{e}}+K_{r}
$$

where $E\left(=m c^{2}\right)$ is the energy of the incident light, $U_{r}\left(=-e^{2} / 4 \pi \varepsilon_{0} r\right)$ is the potential due to electrostatic attraction acting on the electron, $K_{\mathrm{e}}\left(=\frac{1}{2} m_{\mathrm{e}} V^{2}\right)$ is the electron orbital kinetic energy, and $K_{r}\left(=\frac{1}{2} \quad m_{\mathrm{e}} W^{2}\right)$ its radial kinetic energy (related to its radial speed $\left.w\right)$.

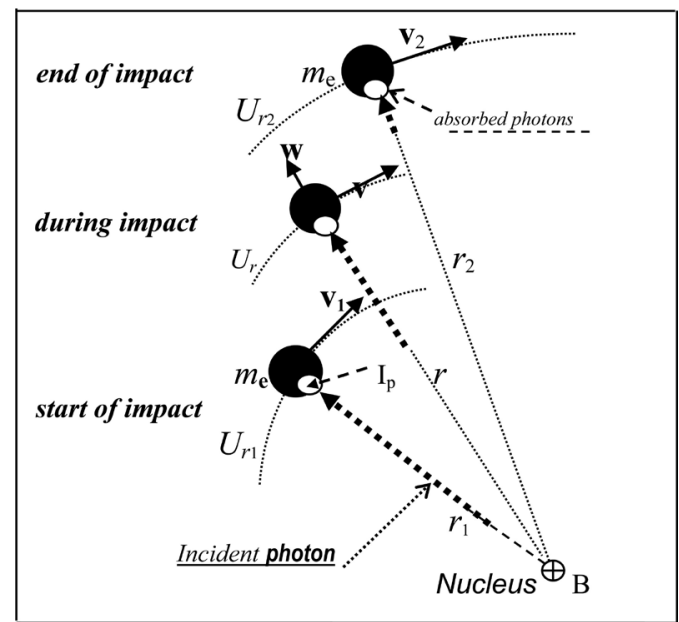

Figure 11. Absorption effect: Incident photons are absorbed by the electron which moves toward higher orbits; when re-emitted, (electron moving toward inner orbits), have contrary direction.

Regarding the Absorption/Emission effect (elements on gaseous form), see Figure 11 , along circular orbits it is $K_{r}=0$ and since, at the end of absorption, along the orbit $r_{2}$, (where the photons have been absorbed), it is $E_{2}=0$, between two circular orbits $r_{1}$ and $r_{2}$, the (92) gives

$$
E_{1}+U_{r 1}+K_{\mathrm{e} 1}=U_{r 2}+K_{\mathrm{e} 2} .
$$

Now, from (53), $U_{r}=-m_{\mathrm{e}} v^{2}$, and since $K_{\mathrm{e}}=1 / 2 m_{\mathrm{e}} v^{2}$, we get $\left(U_{r}+K_{\mathrm{e}}\right)=-\frac{1}{2} m_{\mathrm{e}} v^{2}$ so from (93) we have $E_{1}-\frac{1}{2} m_{\mathrm{e}} v_{1}^{2}=-\frac{1}{2} m_{\mathrm{e}} v_{2}^{2}$; thus, as $E_{1}=h v$, and since $m_{\mathrm{e}} v^{2}=e^{2} / 4 \pi \varepsilon_{0} r$ we find

$$
h v=\frac{1}{2} m_{\mathrm{e}} v_{1}^{2}-\frac{1}{2} m_{\mathrm{e}} v_{2}^{2}=\left(e^{2} / 8 \pi \varepsilon_{0}\right)\left[\left(1 / r_{1}\right)-\left(1 / r_{2}\right)\right]
$$

Then, according to (62) we have $r_{1}=r_{0} n^{2}$ and $r_{2}=r_{0} k^{2}$ (with $k>n$ as $r_{2}>r_{1}$ ), thus

$$
v=\left(e^{2} / 8 \pi \varepsilon_{0} h r_{0}\right)_{0}\left[\left(1 / n^{2}\right)-\left(1 / k^{2}\right)\right]
$$


and plugging the (57) written as $v_{0}=e^{2} / 8 \pi \varepsilon_{0} h r_{0}$, we find

$$
v=v_{0}\left[\left(1 / n^{2}\right)-\left(1 / k^{2}\right)\right]
$$

which is the photons frequency between two circular orbits, where $n$, as showed on Section 3.2, is an integer representing the (progressive) number of each circular orbit, and where $k$ turns out to have the values $k\left(=n+1, n+2, \cdots, n_{\mathrm{i}}\right)$ which is, one by one, the number of the remaining external circular orbits.

Claimed fall of a circling electron into its nucleus: an electrical current emits an electro-magnetic radiation and therefore it is claimed that the circulating electrical charge of an electron should also emit an e.m. radiation yielding the electron, in a short time, to fall into the nucleus; but on our results, a free electron, moving, for instance, along a copper wire under an electrical potential difference, when entering into an atom influence, (at that moment the electron charge will return to face the atom nucleus), will release the necessary photons to reach the atom energy level corresponding to the energy previously received (during the absorption effect). Indeed, along circular orbits, it is $W$ $=0$, therefore the absorption/emission of photons may only start/finish along these orbits, thus the circling electrons are absorbing/emitting photons only between circular orbits, so the e.m. radiation related to an electrical current is due to the emitted photons during their re-entry to an atom; by the way, the photons emission is necessary for the electron not to fall into the nucleus.

\subsection{Photoelectric Effect: Number of Photons Necessary for the Atom Ionization}

Between the electron ground-state orbit $r_{0}$ and its extraction orbit $r \rightarrow \infty$ (intending on microscopic scale), the (92), valid for every interaction light-matter, gives

$$
E+U_{r 0}+K_{\mathrm{e} 0}+K_{r 0}=E^{\prime}+U_{r \rightarrow \infty}+K_{\mathrm{e} \infty}+K_{\mathrm{ae}}
$$

with $E^{\prime}$ the energy of re-emitted light, $w_{\text {ae }}$ the electron radial speed after its extraction, ( $K_{\mathrm{ae}}=\frac{1}{2} m_{\mathrm{e}} w_{\mathrm{ae}}^{2}$ its kinetic energy), while the other terms have been defined referring to (92).

On ground-state, as also shown between Equations ((93) and (94)), it is

$$
U_{r 0}+K_{\mathrm{e} 0}=-\frac{1}{2} m_{\mathrm{e}} v_{0}^{2}\left(=-W_{\mathrm{f}}\right)
$$

with $V_{0}$ the electron speed along $r_{0}$ and with $W_{\mathrm{f}}$ the Work function (electron extraction work); now, at the start of impact, $w=0$ giving $K_{r 0}\left(=\frac{1}{2} m_{\mathrm{e}} w^{2}\right)=0$, while for $r \rightarrow \infty$, the electron orbital speed $v_{\infty} K_{r 0}\left(=\frac{1}{2} m_{\mathrm{e}} w^{2}\right)=0 \rightarrow 0$, so $\left(U_{r \rightarrow \infty}+K_{\mathrm{e} \infty}\right)=-\frac{1}{2} m_{\mathrm{e}} v_{\infty}^{2} \rightarrow 0$, and
(97) gives

$$
E-W_{\mathrm{f}}=E^{\prime}+K_{\mathrm{ae}} \Rightarrow E=E^{\prime}+W_{\mathrm{f}}+K_{\mathrm{ae}} \Rightarrow E=E^{\prime}+K=E^{\prime}+\frac{1}{2} m_{\mathrm{e}} w^{2}
$$

where $W_{\mathrm{f}}+K_{\mathrm{ae}} \equiv K=\frac{1}{2} m_{\mathrm{e}} w^{2}$ is the total kinetic energy transferred from light to electron. On Photoelectric Effect (PhE), the light scatters off an electron $\left(K_{\mathrm{ae}} \geq 0\right)$, but it is not re-emitted, $\left(E^{\prime}=0\right)$, so the $(99)$, with $v_{\mathrm{f}}\left(=W_{\mathrm{f}} / h\right)$ the specific threshold frequency, 
becomes

$$
E=W_{\mathrm{f}}+K_{\mathrm{ae}}=K=\frac{1}{2} m_{\mathrm{e}} w^{2} \Rightarrow h v=h_{\mathrm{f}} v_{\mathrm{f}}+\frac{1}{2} m_{\mathrm{e}} w_{\mathrm{ae}}^{2}=\frac{1}{2} m_{\mathrm{e}} w^{2}
$$

showing that for $v=v_{\mathrm{f}}$ there is ionization with $w_{\mathrm{ae}}=0$.

At frequency $v_{\mathrm{f}}$ the electron radial speed $w_{\mathrm{f}}$, due to the impact of one photon, see Equation (75), is $w_{\mathrm{f}}=2 h v_{\mathrm{f}} / m_{\mathrm{e}} c=2 W_{\mathrm{f}} / m_{\mathrm{e}} c$, and writing the (55) as $v_{0}=\left(2 W_{\mathrm{f}} / m_{\mathrm{e}}\right)^{1 / 2}$, we get

$$
w_{\mathrm{f}} / v_{0}=\left(2 W_{\mathrm{f}} / m_{\mathrm{e}} c\right) / \sqrt{2 W_{\mathrm{f}} / m_{\mathrm{e}}}=\sqrt{2 W_{\mathrm{f}} / m_{\mathrm{e}}} / c=v_{0} / c \Rightarrow w_{\mathrm{f}}=v_{0}^{2} / c
$$

and since the values of $W_{\mathrm{f}}$ are in the range $2-6 \mathrm{eV}$, the (101) gives $W_{\mathrm{f}} / v_{0} \cong 0.0028$ 0.0048 meaning that the ionization, requiring $w=v_{0}$, at frequency $v_{\mathrm{f}}$ needs $n_{\mathrm{f}}$ photons, as follows: the electron radial speed due to $n_{\mathrm{f}}$ photons with frequency $v_{\mathrm{f}}$, see (76), is $w_{n \mathrm{f}}=n_{\mathrm{f}} w_{\mathrm{f}}=n_{\mathrm{f}} 2 W_{\mathrm{f}} / m_{\mathrm{e}} c$, so the ionization condition $(w=v)$ becomes $w_{n \mathrm{f}}=v_{\mathrm{o}}$ leading to $n_{\mathrm{f}} 2 W_{\mathrm{f}} / m_{\mathrm{e}} c=\left(2 W_{\mathrm{f}} / m_{\mathrm{e}}\right)^{1 / 2}$ giving

$$
n_{\mathrm{f}}=c \sqrt{m_{\mathrm{e}} / 2 W_{\mathrm{f}}}=c / v_{0}=v_{0} / w_{\mathrm{f}}
$$

that is, on $\mathrm{PhE}$, the number of photons at frequency $v_{\mathrm{f}}$ necessary for ionization $\left(w_{\mathrm{ae}}=0\right)$. Now, if $n_{\mathrm{f}}$ photons, at frequency $v_{\mathrm{f}}$, are sufficient for ionization, then the frequency

$$
n_{\mathrm{f}} v_{\mathrm{f}} \equiv v_{1}
$$

is sufficient for ionization ( $w_{\mathrm{ae}}=0$ ) with one photon only, meaning that $v_{1}$ is the threshold between $\mathrm{PhE}$ and Compton effect which requires one photon only, as shown on next chapter.

Now let us find the number $n_{1}$ (giving $w_{\text {ae } \max }$ ) of the impacting photons at frequency $v_{1}$ : writing the (100) as $h v=1 / 2 m_{\mathrm{e}} W^{2}$, (energy transferred from a ray of light to an electron), one gets $W=\left(2 h v / m_{\mathrm{e}}\right)^{1 / 2}$ which has to be equal to the electron radial speed due to $n$ photons, $w_{n}=2 n h v / \mathrm{cm}_{\mathrm{e}}$, see (76); so, for $v=v_{1}$ and $n=n_{1}$, we get

$$
\begin{aligned}
\left(2 h v_{1} / m_{\mathrm{e}}\right)^{1 / 2} & =2 n_{1} h v_{1} / c m_{\mathrm{e}} \quad \text { yielding } \\
n_{1}^{2} & =m_{\mathrm{e}} c^{2} / 2 h v_{1}=m_{\mathrm{e}} c^{2} / 2 h n_{\mathrm{f}} v_{\mathrm{f}}=m_{\mathrm{e}} c^{2} / 2 n_{\mathrm{f}} W_{\mathrm{f}} \Rightarrow n_{1}=c \sqrt{m_{\mathrm{e}} / 2 n_{\mathrm{f}} W_{\mathrm{f}}}
\end{aligned}
$$

with $n_{1}$ the number of impacting photons at frequency $v_{1}$ and plugging $n_{\mathrm{f}}$ given by (102), we find

$$
n_{1}=\sqrt{n_{\mathrm{f}}}
$$

meaning that on $\mathrm{PhE}$, the number of impacts photons-electron varies from $n_{\mathrm{f}}$ related to the frequency $v_{\mathrm{f}}$ to $n_{1}\left(=n_{\mathrm{f}}^{1 / 2}\right)$ related to the max admitted frequency $v_{1}\left(=v_{\mathrm{f}} n_{\mathrm{f}}\right)$. For instance as for caesium (Cs), having $W_{\mathrm{f}} \cong 2 \mathrm{eV}$, since $n_{\mathrm{f}}=c\left(m_{\mathrm{e}} / 2 W_{\mathrm{f}}\right)^{1 / 2} \cong 357$, we may infer $n_{\mathrm{f}}=361$ leading to $n_{1}=19$, while as for Pt, having $W_{\mathrm{f}} \cong 6 \mathrm{eV}$, we may infer $n_{\mathrm{f}}=196$ leading to $n_{1}=14$.

\subsection{Compton Effect: Number of Photons Involved and Compton Equation via Doppler Effect}

Here, see Figure 12, the incident photon (length $\lambda$, frequency $v$ ), while ejecting a circling electron is also reflected $(\lambda, v)$ so the recoiling electron, emitting a photon $\lambda^{\prime}$ toward the Observer A, represents a source in motion from A along the direction $\mathbf{w}$, implying an undoubted Doppler effect. 


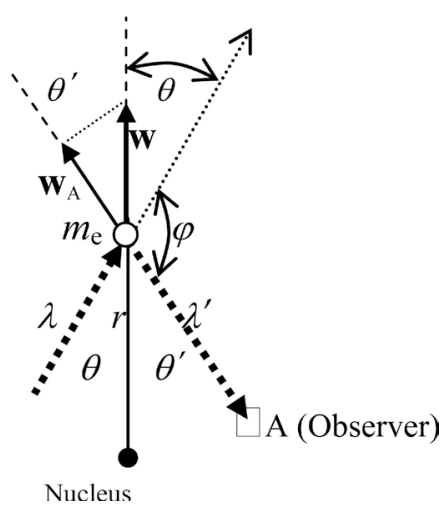

Figure 12. Compton effect (CE). $\varphi$ : angle between the direction of the incident photon and the scattered one $(\lambda) ; \theta$. angle between the direction of the incident photon $\lambda$ and the recoiled electron; $\theta^{\prime}(=\pi-\varphi-\theta)$ : it will be shown that $\theta^{\prime}=\theta$.

Now, on the basis that the scattered photon starts to be reflected at the same time when the incident photon starts to hit the electron, and since $T^{\prime}\left(=1 / v^{\prime}\right)$ is the emission time of the reflected photon, it turns out that $T^{\prime}$ is also the whole interaction time, meaning that there is not a complete absorption of the incident photon followed by an emission. Now, with $T^{\prime}$ the whole impact time photon-electron, the momentum transferred from the incident light to the electron, as per (30), is $p\left(=m^{\prime} c=\gamma v^{\prime} c=\gamma c / T^{\prime}\right)$ and the same value is then transferred from the reflected photon to the electron, so the Conservation of Momentum (CoM) along the direction normal to $\mathbf{w}$, becomes

$$
\gamma c / T^{\prime} \sin \theta=\gamma c / T \sin \theta^{\prime}
$$

giving $\theta=\theta^{\prime}$. Then, the length of the reflected photon, for the Observer A, see Equation (12) is

$$
\lambda^{\prime}=\lambda+\Delta \lambda
$$

where $\Delta \lambda=w_{\mathrm{A}} T^{\prime}$ and where $w_{\mathrm{A}}=w \cos \theta$ is the component of the electron speed along the direction of the Observer A and $T^{\prime}\left(=1 / v^{\prime}\right)$ is, for A, the photon transit time, so we get

$$
\lambda^{\prime}-\lambda(\equiv \Delta \lambda)=w T^{\prime} \cos \theta
$$

Now the CoM along $\mathbf{w}$ is

$$
\left(\gamma c / T^{\prime} \cos \theta\right)+\left(\gamma c / T^{\prime} \cos \theta\right)=m_{\mathrm{e}} w
$$

giving

$$
w T^{\prime}=(2 \gamma c \cos \theta) / m_{\mathrm{e}} .
$$

Then, plugging this value into (108) we get

$$
\lambda^{\prime}-\lambda(\equiv \Delta \lambda)=\left(2 \gamma c \cos ^{2} \theta\right) / m_{\mathrm{e}}=\left(2 h \cos ^{2} \theta\right) / \mathrm{cm}_{\mathrm{e}}
$$

Now, $2 \theta+\varphi=\pi, \Rightarrow \theta=(\pi-\varphi) / 2$, hence $\cos \theta=\sin \varphi / 2$ and therefore

$$
\Delta \lambda=2 h \sin ^{2}\left(\frac{\varphi}{2}\right) / c m_{e}
$$


and $\operatorname{since} 2 \sin ^{2} \frac{\varphi}{2}=(1-\cos \varphi)$, we get the Compton equation:

$$
\Delta \lambda=\lambda^{\prime}-\lambda=h(1-\cos \varphi) / \mathrm{cm}_{\mathrm{e}}
$$

which cannot be obtained via the Doppler effect relativistic equations regarding the light.

Then, the (110), for $\cos \theta=1$, equals the (75), implying the impact of one photon only.

Now, the (111), for $\cos \theta=1$ gives $\lambda^{\prime}=(c / v)+2 \gamma c / m_{\mathrm{e}}$, or

$$
\begin{aligned}
& T^{\prime}=(1 / v)+2 \gamma / m_{\mathrm{e}}=\left(m_{\mathrm{e}}+2 \gamma v\right) / v m_{\mathrm{e}} \text { which plugged into (110) gives } \\
& \qquad w=\left(2 \gamma c / m_{\mathrm{e}}\right) /\left(m_{\mathrm{e}}+2 \gamma v\right) / v m_{\mathrm{e}}=2 \gamma v c /\left(m_{\mathrm{e}}+2 \gamma v\right)=c /\left(1+m_{\mathrm{e}} / 2 \gamma v\right)
\end{aligned}
$$

yielding, see Figure 13,w $\rightarrow c$ for $v \rightarrow \infty$, whereas, for inelastic impacts, $w$ is proportional to $v$.

Then, as for the electron radial speed after its extraction, here indicated $v$, from (100) we have $W_{\mathrm{f}}+K_{\mathrm{ae}}=1 / 2 m_{\mathrm{e}} W^{2}$ where $K_{\mathrm{ae}}=1 / 2 m_{\mathrm{e}} v^{2}$ and since $W_{\mathrm{f}}=\frac{1}{2} m_{\mathrm{ee}} v_{0}^{2}$ as shown by (98), we get

$$
\frac{1}{2} m_{\mathrm{e}} v_{0}^{2}+\frac{1}{2} m_{\mathrm{e}} v^{2}=\frac{1}{2} m_{\mathrm{e}} w^{2} \Rightarrow v=\sqrt{w^{2}-v_{0}^{2}}
$$

which for $w=v_{0}$, (ionization condition) gives $v=0$, as represented on the Figure.

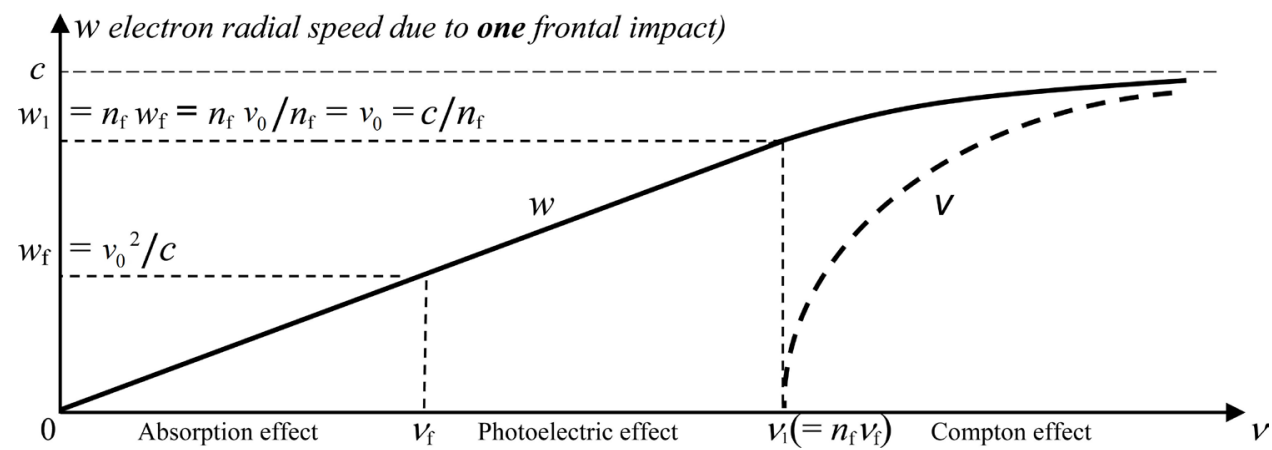

Figure 13. Relation between the incident frequency vand the electron radial speed $(w)$ due to one photon.

\section{Conclusions}

The Relativity arose as a result of attempts to explain the (apparent) constancy of the speed of light which was supported by many experiments: in fact, on our bases/results, there is, on Earth, a continuous variation of $c_{\mathrm{o}}$ ( $c$ on Earth) which, from Equation (8), can be written $\Delta c=-\Delta U / c_{0}$ with $\Delta U$ the variation of the total potential on Earth (mainly due to the variable distance (d) Earth-Sun); indeed, between Aphelion and Perihelion $\left(\Delta d_{\mathrm{PA}} \cong 5 \times 10^{9} \mathrm{~m}\right)$, we get $\Delta c_{P A}=-\Delta U / c_{\mathrm{o}}=-M_{\mathrm{s}} G \Delta d_{\mathrm{PA}} / d^{2} c_{\mathrm{o}} \cong-0.1 \mathrm{~m} \cdot \mathrm{s}^{-1}$ well lower than the accuracy of the measured value of $c_{0}$. Anyhow, under the assumption that the light is composed of longitudinal-extended elastic and massive particles (photons) emitted at a speed equal to the total escape speed, we showed that the speed of light, under a constant total potential, is constant for every Observer, in accordance with the Newtonian laws. 
Moreover, the Relativity Theory may last until a contrary experiment: well, an update experiment, similar to the Harvard tower experiment, would show that the direction of the compensating velocity, (between the source and the absorber), is contrary, as per our results, to the one predicted by the Relativity.

The Quantum Mechanics arose as a result of attempts to explain the discrete spectrum of the hydrogen atom: here, a revised electron structure, an $\mathrm{H}$ atom new configuration, and the introduction of these photons for the interaction light-matter, gave a Newtonian answer to that question.

On the Appendix, we have described, in short, the main differences between the Relativity and our results, as well as between QM and our results.

\section{References}

[1] Bacchieri, A. (2014) Journal of Modern Physics, 5, 884-889. http://dx.doi.org/10.4236/jmp.2014.59092

[2] Pound, R.V. and Rebka Jr., G.A. (1960) Physical Review Letters, 4, 337. http://dx.doi.org/10.1103/PhysRevLett.4.337

[3] Pound, R.V. and Snider, J.L. (1964) Physical ReviewLetters, 13, 539. http://dx.doi.org/10.1103/PhysRevLett.13.539

[4] Pound, R.V. and Snider, J.L. (1965) Physical Review, 140, 788. http://dx.doi.org/10.1103/PhysRev.140.B788

[5] Nasa Extragalactic Database: (i.e. Galaxy M86 Has $z \cong-0.001$ with $s \cong 16 \mathrm{Mpc}$; M99 Has $z$ $\cong+0.008$ with $s \cong 15 \mathrm{Mpc}$; NGC0063 Has $z \cong+0.004$ with $s \cong 20 \mathrm{Mpc}$; VCC0815 Has $z \cong$ -0.0025 with $s \cong 20 \mathrm{Mpc}$ ). 


\section{Appendix}

Comparison sheet, (short summary), between the Relativity Theory and our results.

Abbreviations. $\mathrm{S}=$ source (of light); $v=$ freq. of light stated by S; $\mathrm{O}=$ Observer; $v_{\mathrm{O}}=$ freq. stated by $\mathrm{O} ; U=$ totalgrav. potential.

\section{Subject}

Composition of the light

Frequency of the light

Wavelength

Reason of the value of $c$

Value of $c$

Longitud. Doppler effect

Transverse Doppler effect

$\mathrm{S}$ on the ground emits upwards $\mathrm{S}$ in altitude emitting downwards $S$ taken from ground to altitude $h$

Atomic clocks on GPS satellites Cosmological high red shift (light from far sources to the Earth)

\section{According to Relativity \\ wave/ massless point-particle \\ number of waves in $1 \mathrm{~s}$ \\ distance between two wave peaks \\ unknown \\ constant

$$
\begin{aligned}
& v_{\mathrm{O}}=v(1 \pm \beta) /\left(1 \pm \beta^{2}\right)^{1 / 2} \\
& v_{\mathrm{O}}=v\left(1 \pm \beta^{2}\right)^{1 / 2}
\end{aligned}
$$

$\mathrm{O}$ in altitude $(h)$ states a red shift $\mathrm{O}$ on the ground states a blue shift at height $h, c_{h}=c_{0}, v_{h}=v_{0}, \lambda_{h}=\lambda_{0}$

time dilation $\Delta t=38.4 \mu \mathrm{s} / \mathrm{day}$ due to Doppler effect, implying the universe expansion

\section{Our results}

longitudinal-extended elastic particle (photon). number of photons, same ray, crossing $\mathrm{O}$ in $1 \mathrm{~s}$. length of one photon.

Escape speed from all the masses: $c=(-2 U)^{1 / 2}$. constant if $U$ is constant; $c \rightarrow 0$ for $U \rightarrow 0$.

$$
\begin{aligned}
& v_{\mathrm{O}}=v /(1 \pm \beta) \\
& v_{\mathrm{O}}=v /\left(1 \pm \beta^{2}\right)^{1 / 2}
\end{aligned}
$$

$\mathrm{O}$ in altitude $(h)$ observes a blue shift. $\mathrm{O}$ on the ground states a red shift.

$$
\left|U_{h}\right|<\left|U_{0}\right| \Rightarrow c_{h}<c_{0} ; \quad v_{h}<v_{0} ; \lambda_{h}=\lambda_{0} .
$$

same value, but due to variation of $v_{h}$. due to increase of $c$ (and $\lambda$ too) along the path source-Earth where $\left|U_{\mathrm{E}}\right| \gg\left|U_{\mathrm{S}}\right|$.

\section{between $Q M$ and our results}

Abbreviations: elc: electron; gso: ground state orbit; $v_{\mathrm{e}}$ : elc freq.; $m_{\mathrm{r}}$ : elc reduced mass; $m_{\mathrm{p}}$ : proton mass; $\mathbf{N}$ : atom nucleus; on $\mathbf{H}$ atom: $r_{\mathrm{o}}$ : gso of $m_{\mathrm{r}}$ ref. to $\mathbf{N} ; v_{\mathrm{eo}}$ : freq. of $m_{\mathrm{r}}$ along $r_{\mathrm{o}} ; v_{\mathrm{eB}}$ : freq. of $m_{\mathrm{r}}$ along $r_{\mathrm{B}} ; v_{\mathrm{o}}$ : photon freq. along $r_{\mathrm{o}}$.

\section{Subject}

Structure of electron

as for the $\mathrm{H}$ atom:

Bohr radius, $r_{\mathrm{B}}$

$2 v_{0} / v_{\mathrm{e}}$

$n$ : quantum numbers

Meaning of $n$

$r_{\mathrm{e}}$ : elc radius

$m_{i}$ elc impact mass (new term)

elc radial speed due to photon $v_{\mathrm{o}}$ elc orbital speed along its gso $r_{\mathrm{i}}$ : elc ionization orbit

Photoel effect: num of photons at $v_{\mathrm{f}}$, with $v_{\mathrm{f}}=W_{\mathrm{f}} / h$, the threshold freq.

Compton effect, photons required Photons emitted by a source, power $P$

\section{According to QM}

assumed as a point-particle

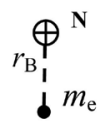

gso of electron (and charge)

$2 v_{0} / v_{\mathrm{eB}}=2 R_{\mathrm{H}} c / v_{\mathrm{eB}}=1.00027$

$$
n=1 \rightarrow \infty
$$

related to the $\mathrm{H}$ atom energy level

$$
=\alpha^{2} r_{\mathrm{B}} \cong 2.82 \mathrm{fm} \text { (known as classical value) }
$$

not predicted

not predicted

$v_{0}=\left(e^{2} / 4 \pi \varepsilon_{0} m_{\mathrm{e}} r_{\mathrm{B}}\right)^{1 / 2}=\alpha c$ $r_{\mathrm{i}}=$ not defined

\section{not predicted \\ $n_{\gamma}=1$ (ray) \\ $n_{\gamma}=P / h v$ photons $/ \mathrm{s}$}

(on our results, it is the number of rays/s)

\section{Our results}

as shown hererafter

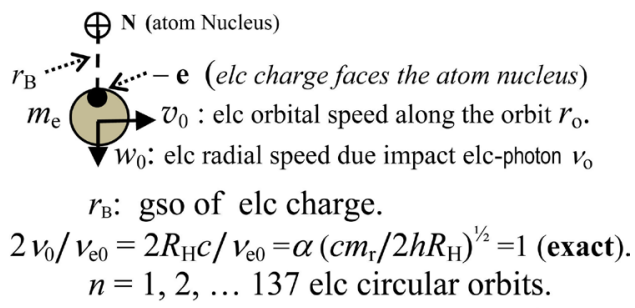

number of photons (same ray) absorbed/emitted during 2 elc circular orbits.

$$
\begin{gathered}
r_{\mathrm{e}}=r_{\mathrm{B}}\left[\alpha^{2} 137^{2}\left(1+m_{\mathrm{e}} / m_{\mathrm{p}}\right)-1\right] \cong 1.005 \mathrm{fm} \\
m_{i} \equiv m_{\mathrm{r}}\left(1+r_{\mathrm{e}} / r_{\mathrm{B}}\right) /\left(1+m_{\mathrm{e}} / m_{\mathrm{p}}\right) . \\
w_{\mathrm{o}}=2 h v_{\mathrm{o}} / c m_{i}=c / 137^{2} . \\
v_{0}=\alpha c\left[\left(1+m_{\mathrm{e}} / m_{\mathrm{p}}\right) /\left(1+r_{\mathrm{e}} / r_{\mathrm{B}}\right)\right]^{1 / 2}=c / 137 \\
r_{\mathrm{i}}=137^{2} r_{\mathrm{o}}
\end{gathered}
$$

$n_{\gamma}=c\left(m_{\mathrm{e}} / 2 W_{\mathrm{f}}\right)^{1 / 2}$ necessary for ionization

$n_{\gamma}=1$ photon, length $\lambda$

$n_{\gamma}=P / h$ photons ( emitted in $1 \mathrm{~s}$ ), all the rays 


\section{Symbols}

$E\left(=m c^{2}\right)$ energy of the light flowing along one ray,

$m=$ mass of light passing along one ray in $1 \mathrm{~s}$,

$v=$ photons frequency (number of photons of the same ray, crossing an Observer, in 1s),

$T=$ photon transit time (time for one photon to cross an Observer),

$\gamma(=m T)$ mass of light passing along one ray during $T$,

$v_{0}=$ photon admitted frequency along the electron ground-state orbit, on $\mathrm{H}$ atom

$v_{n}=$ photon admitted frequency along the $\mathrm{n}^{\text {th }}$ circular electron orbit on $\mathrm{H}$ atom,

$v_{\mathrm{f}}\left(=W_{\mathrm{f}} / h\right)$ : specific threshold frequency on Photoelectric effect $(\mathrm{PhE})$,

$v_{1}=\max$ admitted frequency on $\mathrm{PhE}$; also minimum frequency able to produce the Compton effect,

$\mathcal{E}_{\mathrm{m}} \equiv m_{\mathrm{e}} / m_{\mathrm{P}}$ (where $m_{\mathrm{e}}=$ electron mass and $m_{\mathrm{p}}=$ proton mass),

$r_{0}=$ ground-state electron orbit on $\mathrm{H}$ atom: orbit of $m_{\mathrm{r}}$ referred to $m_{\mathrm{p}}$ (see Figure 8),

$r_{\mathrm{B}}=$ Bohr radius: electron charge orbit, referred to common centre of gravity (CCG)

electron-proton,

$r_{\mathrm{B}}^{\prime}=$ adjusted Bohr radius: electron centre of mass referred to the CCG electron-proton,

$\mathcal{E}_{\mathrm{r}} \equiv r_{\mathrm{e}} / r_{\mathrm{B}}$ (where $r_{\mathrm{e}}=$ electron radius),

$v_{\mathrm{e}}=$ electron frequency,

$v_{\mathrm{e} 0}=$ electron frequency on ground-state orbit $r_{0}, \mathrm{H}$ atom,

$v_{\mathrm{e} n}=$ electron frequency along the $\mathrm{n}^{\text {th }}$ orbit, $\mathrm{H}$ atom,

$V=$ generic speed, also electron orbital speed,

$w=$ electron radial speed, due to the impact of one photon, referred to the atom centre

of gravity,

$w_{0}=$ electron radial speed due to the impact of one photon with frequency $v_{0}$,

$w_{\mathrm{f}}=$ electron radial speed due to the impact of one photon with frequency $v_{\mathrm{f}}$,

$W_{n}=$ electron radial speed due to the impact of $n$ photons,

$W_{n 0}=$ electron radial speed due to the impact of $n$ photons with frequency $v_{0}$,

$n_{\mathrm{i}}=$ number of admitted photons along the ionization orbit,

$w_{\text {ni }}=$ electron radial speed due to the impact of $n$ photons with frequency $v_{0} / n_{\mathrm{i}}^{2}$,

$w_{n^{2}}=$ electron radial speed due to the impact of $n^{2}$ photons,

$K_{\mathrm{r}}\left(=\frac{1}{2} m_{\mathrm{e}} w^{2}\right)=$ electron radial kinetic energy,

$W_{\mathrm{ae}}=$ electron radial speed after extraction (on macroscopic scale), on photoelectric ef-

fect $(\mathrm{PhE})$,

$K_{\mathrm{ae}}=$ electron radial kinetic energy after extraction (on macroscopic scale), on $\mathrm{PhE}$,

$v=$ electron radial speed after extraction (on macroscopic scale), on Compton effect. 
Submit or recommend next manuscript to SCIRP and we will provide best service for you:

Accepting pre-submission inquiries through Email, Facebook, LinkedIn, Twitter, etc. A wide selection of journals (inclusive of 9 subjects, more than 200 journals)

Providing 24-hour high-quality service

User-friendly online submission system

Fair and swift peer-review system

Efficient typesetting and proofreading procedure

Display of the result of downloads and visits, as well as the number of cited articles

Maximum dissemination of your research work

Submit your manuscript at: http://papersubmission.scirp.org/

Or contact jmp@scirp.org 